九州大学学術情報リポジトリ

Kyushu University Institutional Repository

\title{
A Finite Element Scheme for Two-Layer Viscous Shallow-Water Equations
}

Kanayama, Hiroshi

Department of Intelligent Machinery and Systems Faculty of Engineering, Kyushu University

Dan, Hiroshi

Department of Intelligent Machinery and Systems Faculty of Engineering, Kyushu University

http://hdl. handle. net/2324/5552

出版情報: Japan Journal of Industrial and Applied Mathematics. 23 (2), pp.163-191，2006-06. Kinokuniya

バージョン:

権利関係：๑2006 日本応用数理学会 


\section{A Finite Element Scheme for}

\section{Two-Layer Viscous Shallow-Water Equations}

Hiroshi KANAYAMA* and Hiroshi DAN*

*Faculty of Engineering, Kyushu University,

Fukuoka 819-0395, Japan

E-mail: \{kanayama,dan\}@mech.kyushu-u.ac.jp 
In this paper, the two-layer viscous shallow-water equations are derived from the three-dimensional Navier-Stokes equations under the hydrostatic assumption. It is noted that the combination of upper and lower equations in the two-layer model produces the classical one-layer equations if the density of each layer is the same. The two-layer equations are approximated by a finite element method which follows our numerical scheme established for the one-layer model in 1978. Finally, it is numerically demonstrated that the interfacial instability generated when the densities are the same can be eliminated by providing a sufficient density difference.

Key words: Shallow-water, Layer model, Navier-Stokes equations, Finite element scheme 


\section{Introduction}

This research focuses on shallow-water equations produced using the layer model [9], [10], which is a well-known technique for quasi-three-dimensional computations. The research attempts to generate a computational method using finite element techniques based on the previous investigations of Kanayama and Ohtsuka (1978) [4]. First, a two-layer shallow-water equation system is derived from the Navier-Stokes equations, based on the assumption of hydrostatic pressure in the direction of gravity. Next, it is noted that when the upper-layer and lower-layer densities are the same, combination of the equations produces an equation system that is identical to the one-layer equations [5]. Finally, results of one-layer and two-layer computations are presented, and it is numerically demonstrated that the interfacial instability [9] generated when the densities are the same can be eliminated by providing a sufficient density difference.

The layer model is useful when the density of each layer is different and two layers are stratified. Some examples of such a case are stratification of the sea water in some season, stratification due to oil spreading by accidents and others. Our main concern in this paper is to remodel such a two layer sea flow by using the layer model and to produce a suitable numerical method to compute the phenomena by using the finite element method. Though the two layer model is well known [9], [10], we reconstruct the model so that the combination of each layer equations produces the classical one layer equation system [5]. Appendixes 1 and 2 are added for completeness and self-containment. Appendix 1 shows how the two-layer shallow-water system is derived. Appendix 2 also shows details of combination when the upper-layer and lower-layer densities are the same. Then finite element approximations of two layer equations are performed following our previous work [4] for the one layer equations. To show the validity of our approach, we pick up two concrete examples. One is the wind-driven flow in Mikawa Bay for which one layer computation was performed in [2]. The other is concerned with the Ariake Sea which several researchers are trying to study from some view points because of recent environmental problems. In this paper, we also consider the wind-driven flow in the Ariake Sea because our main concern of numerical experiments is whether the interfacial instability observed in [9] still occurs in our 
reformulated two layer model and also it is overcome by a density difference or not. Since observed data are not got easily, we show results of a simple physical experiment in Fig. 4, which demonstrate that stratification of the two layer model really happens if a sufficient condition is given. Appendix 3 explains a similar numerical experiment to the above physical one and again shows that the interfacial instability still occurs and that it is overcome by a sufficient density difference. It may be very surprising that combination of the equations produces an equation system that is identical to the one-layer equations when the upper-layer and lower-layer densities are the same, but that the two-layer shallow-water system is then difficult to solve numerically because of the interfacial instability. So, this a little contradictious point may be one of our original claims. The interfacial instability firstly and shortly appeared in Murakami (1989) [9] in which the instability was simply explained as one of numerical instabilities without detail descriptions. The present authors strongly insist that this numerical instability inherently comes from the two-layer model and not from numerical schemes like our finite element scheme. Since the interface of the two-layer model is quite artificial when the upper-layer and lower-layer densities are the same, it may be natural to be removed quickly in computation. So, the one-layer model should be used in the absence of the density difference. When the density difference is not so large, numerical computations also become difficult. The remedy remains an open problem.

\section{Derivation of two-layer viscous shallow-water equations}

The physical domain is divided into two layers, as shown in Fig. 1. It is assumed that the horizontal length scale is much larger than water depth, and that there is the shallow-water low-frequency flow. First, two-layer viscous shallow-water equations will be derived. As shown in Fig. 1, orthogonal coordinates $[m]$ are used wherein $x_{1}$ and $x_{2}$ represent directions in the horizontal plane, and $x_{3}$ represents the normal direction. Time $[s]$ is represented by $t$. Assuming hydrostatic pressure in the $x_{3}$ direction, the system is formulated using the Navier-Stokes equations, with Coriolis forces occurring in the $x_{1}$ and $x_{2}$ directions and gravity acting in the $x_{3}$ direction. 


$$
\begin{aligned}
& \sum_{j=1}^{3} \frac{\partial}{\partial x_{j}} u_{j}=0, \\
& \frac{\partial u_{1}}{\partial t}+\sum_{j=1}^{3} u_{j} \frac{\partial u_{1}}{\partial x_{j}}=-\frac{1}{\rho} \frac{\partial p}{\partial x_{1}}+\frac{1}{\rho} \sum_{j=1}^{3} \frac{\partial}{\partial x_{j}} \tau_{1 j}+f u_{2}, \\
& \frac{\partial u_{2}}{\partial t}+\sum_{j=1}^{3} u_{j} \frac{\partial u_{2}}{\partial x_{j}}=-\frac{1}{\rho} \frac{\partial p}{\partial x_{2}}+\frac{1}{\rho} \sum_{j=1}^{3} \frac{\partial}{\partial x_{j}} \tau_{2 j}-f u_{1}, \\
& 0=-\frac{1}{\rho} \frac{\partial p}{\partial x_{3}}-g .
\end{aligned}
$$

Fig. 1. 2 layer model

$u\left(x_{1}, x_{2}, x_{3}, t\right)$ represents velocity $[\mathrm{m} / \mathrm{s}]$ in the $x_{i}(i=1-3)$ direction, $p\left(x_{1}, x_{2}, x_{3}, t\right)$ denotes pressure $\left[N / m^{2}\right], \rho$ denotes density $\left[\mathrm{kg} / \mathrm{m}^{3}\right], \tau_{i j}$ denotes stress $\left[N / \mathrm{m}^{2}\right]$ in the direction $x_{i}$ acting on the $x_{j}$ plane, $f$ denotes the Coriolis coefficient $[1 / \mathrm{s}]$ and $g$ denotes acceleration $\left[\mathrm{m} / \mathrm{s}^{2}\right]$ due to gravity. In addition, the vertical displacement of the water's free surface is represented by the ordinate $\zeta$, the vertical displacement of the interface is represented by the ordinate $d$, and the ordinate representing the bottom boundary surface of the water is represented by $b$. On the water's free surface plane, interfacial plane and water bottom plane, there is no velocity component in the normal direction, and so the following conditions are used for $u_{3}$.

$$
\left\{\begin{array}{l}
u_{3}=\frac{\partial \zeta}{\partial t}+\sum_{j=1}^{2} u_{j} \frac{\partial \zeta}{\partial x_{j}}\left(x_{3}=\zeta\left(x_{1}, x_{2}, t\right)\right), \\
u_{3}=\frac{\partial d}{\partial t}+\sum_{j=1}^{2} u_{j} \frac{\partial d}{\partial x_{j}}\left(x_{3}=d\left(x_{1}, x_{2}, t\right)\right), \\
u_{3}=\sum_{j=1}^{2} u_{j} \frac{\partial b}{\partial x_{j}} \quad\left(x_{3}=b\left(x_{1}, x_{2}\right)\right) .
\end{array}\right.
$$


We integrate with respect to $x_{3}$ each of the equations (2-1), (2-2) and (2-3), from the ocean floor $b$ to the interface $d$, and from the interface $d$ to the water surface $\zeta$. Then, the two-layer viscous shallow water equations are derived that express averaged rates for each layer. As shown in Fig. 1, the upper layer is denoted the $u$ layer while the lower layer is denoted the $l$ layer. Thus, the velocity component in the $x_{i}(i=1,2)$ direction averaged over the layer $m(m=u, l)$ is taken as $U_{i}^{m}\left(x_{1}, x_{2}, t\right)$. The thickness of the $m$ layer is denoted by $H^{m}\left(x_{1}, x_{2}, t\right)$. We consider fluids of different densities in the two layers, with the constant density in the $m$ layer represented by $\rho^{m}$. $H^{m}$ and $U_{i}^{m}$ are represented by the following equations;

$$
\begin{aligned}
& H^{l}=d-b, \quad H^{u}=\zeta-d, \\
& U_{i}^{l}=\frac{1}{H^{l}} \int_{b}^{d} u_{i} d x_{3}, U_{i}^{u}=\frac{1}{H^{u}} \int_{d}^{\zeta} u_{i} d x_{3} .
\end{aligned}
$$

In addition, stress in the $x_{i}$ direction acing on the $x_{j}$ surface of the $m$ layer is represented by $\tilde{\tau}_{i j}^{m}$, stress in the $x_{i}$ direction acting on the top surface of the $m$ layer is represented by $S_{i}^{m}$, and stress in the $x_{i}$ direction acting on the bottom surface is represented by $B_{i}{ }^{m}$. The horizontal viscosity constant $\left[N s / m^{2}\right]$ is represented by $\mu_{H}$, the wind effect constant is represented by $\theta$, the air density is represented by $\rho_{a}$, the wind speed in the $x_{i}$ direction is represented by $W_{i}$, the Chezy constant $\left[\mathrm{m}^{1 / 2} / \mathrm{s}\right]$ is represented by $C$, and the viscosity constant in the vertical direction is represented by $\mu$. The following two-layer viscous shallow-water equations are thus derived [3] (see also Appendix 1 for completeness and self-containment).

$$
\begin{aligned}
& \frac{\partial}{\partial t} H^{m}+\sum_{j=1}^{2} \frac{\partial}{\partial x_{j}}\left(H^{m} U_{j}^{m}\right)=0 . \\
& H^{m}\left(\frac{\partial}{\partial t} U_{i}^{m}+\sum_{j=1}^{2} U_{j}^{m} \frac{\partial}{\partial x_{j}} U_{i}^{m}\right) \\
& =-g H^{m}\left(\frac{\rho^{u}}{\rho^{m}} \frac{\partial H^{u}}{\partial x_{i}}+\frac{\partial H^{l}}{\partial x_{i}}+\frac{\partial b}{\partial x_{i}}\right)+\frac{1}{\rho^{m}} \sum_{j=1}^{2} \frac{\partial}{\partial x_{j}}\left(H^{m} \tilde{\tau}_{i j}^{m}\right)+\frac{1}{\rho^{m}} S_{i}^{m}-\frac{1}{\rho^{m}} B_{i}^{m}+(-1)^{i+1} f H^{m} U_{i+1}^{m} .
\end{aligned}
$$


In the above, $\quad S_{i}^{u}=\theta \rho_{a} W_{i}\left(\left(W_{1}\right)^{2}+\left(W_{2}\right)^{2}\right)^{\frac{1}{2}}, \quad B_{i}^{l}=\frac{\rho^{l} g}{C^{2}} U_{i}\left(\left(U_{1}\right)^{2}+\left(U_{2}\right)^{2}\right)^{\frac{1}{2}}$,

$$
\begin{aligned}
& B_{i}^{u}=S_{i}^{l}=\frac{2 \mu\left(U_{i}^{u}-U_{i}^{l}\right)}{H^{u}+H^{l}}, \tilde{\tau}_{i j}^{m}=\mu_{H}\left(\frac{\partial U_{i}^{m}}{\partial x_{j}}+\frac{\partial U_{j}^{m}}{\partial x_{i}}\right), U_{i}=\frac{H^{u} U_{i}^{u}+H^{l} U_{i}^{l}}{H^{u}+H^{l}}, \\
& U_{3}^{m}=U_{1}^{m} .
\end{aligned}
$$

\section{Comparison between one-layer and two-layer viscous shallow-water equations}

The two-layer viscous shallow-water equations correspond to the one-layer viscous shallow-water equations when the densities of the upper layer and lower layer are the same $\left(\rho=\rho^{l}=\rho^{u}\right)$ and the upper layer and lower layer equations are combined. The one-layer equation system [5] is presented below.

$$
\begin{aligned}
& \frac{\partial}{\partial t} \zeta+\sum_{j=1}^{2} \frac{\partial}{\partial x_{j}}\left(H U_{j}\right)=0 . \\
& H\left(\frac{\partial}{\partial t} U_{i}+\sum_{j=1}^{2} U_{j} \frac{\partial}{\partial x_{j}} U_{i}\right) \\
& =-g H \frac{\partial \zeta}{\partial x_{i}}+\frac{1}{\rho}\left(\sum_{j=1}^{2} \frac{\partial}{\partial x_{j}}\left(H \tilde{\tau}_{i j}\right)\right)+\frac{\theta \rho_{a}}{\rho} W_{i}\left(\left(W_{1}\right)^{2}+\left(W_{2}\right)^{2}\right)^{\frac{1}{2}}-\frac{g}{C^{2}} U_{i}\left(\left(U_{1}\right)^{2}+\left(U_{2}\right)^{2}\right)^{\frac{1}{2}} \\
& +(-1)^{i+1} f H U_{i+1} .
\end{aligned}
$$

where

$$
\begin{aligned}
& H=H^{u}+H^{l}, \quad U_{i}=\frac{1}{H} \int_{b}^{\zeta} u_{i} d x_{3}=\frac{H^{u} U_{i}^{u}+H^{l} U_{i}^{l}}{H^{u}+H^{l}}, \\
& \tilde{\tau}_{i j}=\mu_{H}\left(\frac{\partial U_{i}}{\partial x_{j}}+\frac{\partial U_{j}}{\partial x_{i}}\right), \quad U_{3}=U_{1} .
\end{aligned}
$$


(3-1) is produced by combining the $u$ form of (2-6) with the $l$ form of (2-6). In fact, when the $u$ form of (2-6) and the $l$ form of (2-6) are added, (3-1) is produced as follows;

$$
\begin{gathered}
\frac{\partial}{\partial t}(\zeta-d)+\frac{\partial}{\partial t} d+\sum_{j=1}^{2} \frac{\partial}{\partial x_{j}}\left(H^{u} U_{j}^{u}\right)+\sum_{j=1}^{2} \frac{\partial}{\partial x_{j}}\left(H^{l} U_{j}^{l}\right) \\
=\frac{\partial}{\partial t} \zeta+\sum_{j=1}^{2} \frac{\partial}{\partial x_{j}}\left(H^{u} U_{j}^{u}+H^{l} U_{j}^{l}\right) \\
=\frac{\partial}{\partial t} \zeta+\sum_{j=1}^{2} \frac{\partial}{\partial x_{j}}\left(H U_{j}\right)=0 .
\end{gathered}
$$

The sum of the $u$ layer and $l$ layer forms of (2-7) can also produce (3-2) in the sense of Appendix 2, using a similar addition. For details refer to the cited literature [3] (see also Appendix 2 for completeness and self-containment).

\section{Finite element approximations for two-layer viscous shallow-water equations}

A finite element approximation for the two-layer viscous shallow-water equations (2-6) and (2-7) in conjunction with appropriate initial and boundary conditions is performed. The computational domain is the two-dimensional polygonal region $\Omega$ surrounded by boundaries $\Gamma_{1}$ and $\Gamma_{2}$. In this region, the orthogonal coordinates $x=\left(x_{1}, x_{2}\right)$ are used. The boundary conditions on $\Gamma_{1}$ and $\Gamma_{2}$, and initial conditions at $t=0$ are presented below.

$$
\begin{aligned}
& \frac{\partial}{\partial t} H^{m}+\sum_{j=1}^{2} \frac{\partial}{\partial x_{j}}\left(H^{m} U_{j}^{m}\right)=0 . \\
& H^{m}\left(\frac{\partial}{\partial t} U_{i}^{m}+\sum_{j=1}^{2} U_{j}^{m} \frac{\partial}{\partial x_{j}} U_{i}^{m}\right) \\
& =-g H^{m}\left(\frac{\rho^{u}}{\rho^{m}} \frac{\partial H^{u}}{\partial x_{i}}+\frac{\partial H^{l}}{\partial x_{i}}+\frac{\partial b}{\partial x_{i}}\right)+\frac{1}{\rho^{m}} \sum_{j=1}^{2} \frac{\partial}{\partial x_{j}}\left(H^{m} \tilde{\tau}_{i j}^{m}\right)+\frac{1}{\rho^{m}} S_{i}^{m}-\frac{1}{\rho^{m}} B_{i}^{m}+(-1)^{i+1} f H^{m} U_{i+1}^{m} .
\end{aligned}
$$


Boundary conditions: $\quad U_{i}^{m}(x, t)=0 \quad$ on $\Gamma_{1}$,

$$
\left.\begin{array}{c}
H^{m}(x, t)=H_{\Gamma_{2}}^{m}(x, t) \\
\sum_{j=1}^{2} \tilde{\tau}_{i j}^{m} n_{j}=0
\end{array}\right\} \quad \text { on } \Gamma_{2} .
$$

$n_{i}$ denotes the $x_{\mathrm{i}}$ component of the unit-normal vector with respect to the boundary.

Initial conditions: $\quad U_{i}^{m}(x, 0)=U_{i 0}^{m}(x), H^{m}(x, 0)=H_{0}^{m}(x)$.

$H_{\Gamma_{2}}^{m}(x, t)$ is specified on $\Gamma_{2}$. The initial value $H_{0}^{m}(x)$ is specified depending on the sea floor topography $b$ and where the layers are initially delimited. $U_{i 0}^{m}(x)$ is the initial value for $U_{i}^{m}$.

The finite element approximation is as follows. First, the respective test functions are assigned for (4-1) and (4-2), and integration is performed over the computational domain $\Omega$. Next, approximation is carried out using an explicit method for terms involving a derivative with respect to time. The finite element approximation [4] is carried out using the basis function $\hat{\varphi}_{k}$ of a piecewise-linear triangulation for terms including spatial derivatives, and using the step function $\bar{\varphi}_{k}$ for terms without spatial derivatives. The step function is 1 in the barycentric region around node $k$, and is 0 at other places.

$$
\begin{gathered}
\left(\frac{\bar{H}^{m, n+1}-\bar{H}^{m, n}}{\Delta t}, \bar{\varphi}_{k}\right)+\left(\sum_{j=1}^{2} \frac{\partial\left(\hat{H}^{m, n} \hat{U}_{j}^{m, n}\right)}{\partial x_{j}}, \hat{\varphi}_{k}\right)=0 . \\
\left(\bar{H}^{m, n+1}\left(\frac{\bar{U}_{i}^{m, n+1}-\bar{U}_{i}^{m, n}}{\Delta t}\right), \bar{\varphi}_{k}\right)+\left(\hat{H}^{m, n+1} \sum_{j=1}^{2} \hat{U}_{j}^{m, n} \frac{\partial \hat{U}_{i}^{m, n}}{\partial x_{j}}, \hat{\varphi}_{k}\right) \\
=-\left(g \hat{H}^{m, n+1}\left(\frac{\rho^{u}}{\rho^{m}} \frac{\partial \hat{H}^{u, n+1}}{\partial x_{i}}+\frac{\partial \hat{H}^{l, n+1}}{\partial x_{i}}+\frac{\partial b}{\partial x_{i}}\right), \hat{\varphi}_{k}\right)-\sum_{j=1}^{2}\left(\frac{1}{\rho^{m}} \hat{H}^{m, n+1} \mu_{H}\left(\frac{\partial \hat{U}_{i}^{m, n}}{\partial x_{j}}+\frac{\partial \hat{U}_{j}^{m, n}}{\partial x_{i}}\right), \frac{\partial \hat{\varphi}_{k}}{\partial x_{j}}\right) \\
+\left(\frac{1}{\rho^{m}} \bar{S}_{i}^{m, n}, \bar{\varphi}_{k}\right)-\left(\frac{1}{\rho^{m}} \bar{B}_{i}^{m, n}, \bar{\varphi}_{k}\right)+\left((-1)^{i+1} f \bar{H}^{m, n+1} \bar{U}_{i+1}^{m, n}, \bar{\varphi}_{k}\right) .
\end{gathered}
$$


In the above, $d_{k}^{n+1}$ and $\zeta_{k}^{n+1}$ are determined from (4-3), and $U_{i, k}^{m, n+1}$ is computed from these values and $U_{i, k}^{m, n}$ using (4-4). In this computation, $d_{k}^{n}, \zeta_{k}^{n}$ and $U_{i, k}^{m, n}$ are, respectively, approximate values for $d(x, t)$, $\zeta(x, t)$ and $U_{i}^{m}(x, t)$ at node $k$ after $\mathrm{n}$ time steps. In addition, $\Delta t$ represents the size of time step. Symbols are defined below.

$$
\left(\frac{\partial U_{i}^{m}}{\partial x_{j}}, v\right)=\int_{\Omega} \frac{\partial U_{i}^{m}}{\partial x_{j}} v d x, \quad \hat{U}_{i}^{m, n}=\sum_{k} U_{i, k}^{m, n} \hat{\varphi}_{k}, \quad \bar{U}_{i}^{m, n}=\sum_{k} U_{i, k}^{m, n} \bar{\varphi}_{k} .
$$

Based on essential boundary conditions, (4-3) is produced for nodes excluding those on $\Gamma_{2}$, and (4-4) is produced for nodes excluding those on $\Gamma_{1}$. On the boundary $\Gamma_{2}$, the upwind approximations of Tabata (1977) [4], [11] are used in the numerical approximation for the advection term of (4-4).

Note 1: For purposes of simplification, $H_{k}^{m, n+1}$ appears as an unknown function in (4-3), but actually, $H_{k}^{m, n+1}$ is computed by determining $\zeta_{k}^{n+1}$ and $d_{k}^{n+1}$. In this case, a value for $d_{k}^{n+1}$ is necessary when $\zeta_{k}^{n+1}$ is determined, and (4-3) for the $l$ layer is computed prior to (4-3) for the $u$ layer.

Note 2: Numerical justification for the approximation scheme for the linearized equations related to the scheme for the one layer equations in [4] appears in Kanayama-Ushijima (1988, 1989) [6], [7].

\section{Computational examples}

Results of computations for the Ariake Sea [1], [8] under a northerly wind, and Mikawa Bay [2] under a northwesterly wind are presented with and without a density difference.

\section{1) Results of computation for a wind-driven flow in Mikawa Bay}

Computation was carried out with Coriolis coefficient $f=2 \omega \sin \phi$, planetary angular velocity $\omega=0.00416[\mathrm{deg} / s]$, latitude $\phi=35^{\circ}$, horizontal viscosity coefficient $\mu_{H}=10,000\left[\mathrm{Ns} / \mathrm{m}^{2}\right]$, wind effect factor $\theta=0.0026$, wind speed 
$W_{1}=2.45[\mathrm{~m} / \mathrm{s}], W_{2}=-5.91[\mathrm{~m} / \mathrm{s}]$, Chezy coefficient $C=61.4\left[\mathrm{~m}^{1 / 2} / \mathrm{s}\right]$, and time step size $\Delta t=1[\mathrm{~s}]$. Regarding mesh data, the total node number is 426 , and the total element number is 729 . Triangular elements are used, where the minimum perpendicular length is $760[\mathrm{~m}]$ and the maximum triangular element side length is $1771[\mathrm{~m}]$. In order to simplify the problem, an average is used for the ocean floor ordinate, thus $b$ is constant with value selected to be at $-10[m]$. Initial conditions are given by $U_{i 0}^{m}(x)=0$ and $H_{0}^{m}(x)=-b / 2$, and boundary conditions by $H_{\Gamma_{2}}^{m}(x, t)=H_{0}^{m}(x)$. With these parameters, results are presented below for a case where the vertical viscosity coefficient $\mu$ is $10,000\left[\mathrm{Ns} / \mathrm{m}^{2}\right]$ and the density $\rho^{l}=\rho^{u}$ is $1000\left[\mathrm{~kg} / \mathrm{m}^{3}\right]$ (Cond. 1 ), and for a case where $\mu=10\left[\mathrm{Ns} / \mathrm{m}^{2}\right], \rho^{u}=950\left[\mathrm{~kg} / \mathrm{m}^{3}\right]$ and $\rho^{l}=1050\left[\mathrm{~kg} / \mathrm{m}^{3}\right]$ (Cond. 2). It is noted that the viscosity constant in the vertical direction is fairly larger even in Cond. 2 than the value adopted in [1] though the horizontal viscosity constant has the same order. In Cond. 1 without the density difference, the constant $\mu$ is , for simplicity, taken as the same as the constant $\mu_{H}$ since we have the one-layer system (3-1) and (3-2) where $\mu$ does not appear explicitly if the two-layer system (2-6) and (2-7) is combined. In 5.2, we will again consider the effect of the value $\mu$. Also, the density difference is a little larger compared with that between the sea water and the natural water. The reason why such a big density difference is adopted will be mentioned at the last part of this chapter.

The results of one-layer and two-layer computations under Cond. 1 are compared. Figure 2(a) is a graph showing $0.004[\mathrm{~m}]$ contour lines for the water level $\zeta$ after 75 hours. The water level contour lines are found to be nearly perpendicular with respect to the wind direction. Similar analysis was carried out for a one-layer scheme as well, and similar results were obtained. Figure 2(b) shows the evolution in time of the water level $\zeta$ from its initial value at three points indicated in Fig. 2(a). The plots are only visible for these three points, and the one-layer results and two-layer results are found to be very similar. Figure 2(c) is a graph showing change in the interface $d$ over time from the initial values. The values give an unstable interface under Cond. 1. Figure 2(d) is a graph showing the evolution in time of the interface $d$ from initial values for Cond. 1 and Cond. 2 at some locations. The computation time is lengthened relative to that of Fig. 2(c). The values at nodes M1(2) and M5(2) are stable under Cond. 2, but those at M1(1) and M5(1) are unstable under Cond. 1. Although the values at M2(1) and M2(2) are both stable, a large discrepancy between these two values is found. Finally, it is also noted 
that under Cond. 2, the interface $d$ is lower under the wind while the water level $\zeta$ is higher under the wind [3].

Fig. 2. (a) Contour map of $\zeta$ after 75 hours (Cond. 1)

(b) Transition of $\zeta$ (Cond. 1)

(c) Transition of $\left(d+H_{0}^{u}\right)$ (Cond. 1)

(d) Transition of $\left(d+H_{0}^{u}\right)$ (Cond. 1 and Cond. 2)

\section{2) Results of computation for a wind-driven flow in the Ariake Sea}

Numerical results are also presented for the Ariake Sea; without a density difference (Cond. 3), and with a density difference (Cond. 4). Computations were carried out with wind speed $W_{1}=0[\mathrm{~m} / \mathrm{s}], W_{2}=-10[\mathrm{~m} / \mathrm{s}]$, total node number $=3907$, total element number $=7326$, triangular element average side length $=1000[m]$, and ocean floor coordinates $b=-60$ to $-10[m]$. When not specifically defined, the same values as for Mikawa Bay were used for physical values, and initial and boundary conditions. Under these parameters, results are presented below for the vertical viscosity coefficient $\mu=10,000\left[\mathrm{Ns} / \mathrm{m}^{2}\right]$, density $\rho^{l}=\rho^{u}=1000\left[\mathrm{~kg} / \mathrm{m}^{3}\right]$, initial conditions $H_{0}^{m}(x)=-b / 2$ (Cond. 3), and $\mu=10\left[N \mathrm{~s} / \mathrm{m}^{2}\right]$, density $\rho^{u}=1000\left[\mathrm{~kg} / \mathrm{m}^{3}\right], \rho^{l}=1100\left[\mathrm{~kg} / \mathrm{m}^{3}\right]$, initial conditions $H_{0}^{u}(x)=7.5[m]$ and $H_{0}^{l}(x)=-7.5-b[m]$ (Cond. 4). To cover the gap between Cond. 3 and Cond. 4, we may consider Cond. 3a which adopts $\mu=10\left[\mathrm{Ns} / \mathrm{m}^{2}\right]$, density $\rho^{l}=\rho^{u}=1000\left[\mathrm{~kg} / \mathrm{m}^{3}\right]$ and initial conditions $H_{0}^{u}(x)=7.5[m]$ and $H_{0}^{l}(x)=-7.5-b[m]$. However, it is noted that computation under Cond. 3a stops at an early stage because the interface $d$ becomes unstable quickly.

The results of one-layer and two-layer computations are compared under Cond. 3. Figure 3(a) is a diagram showing $0.014[\mathrm{~m}]$ contour lines for the water level $\zeta$ after 55 hours. It is clear that the water level contour lines are nearly perpendicular with respect to the wind direction. Figure 3(b) is a graph showing the evolution in time of the water level $\zeta$ from initial conditions for the four points indicated in Fig. 3(a). As with the Mikawa Bay graph, only four points can be seen, and it is found that one-layer results and two-layer results are nearly identical. Figure 3(c) is a graph showing $0.02[\mathrm{~m}]$ contour lines after 55 hours, starting from the initial value for the interface 
d. A mottled form is presented, and thus the interface $d$ is found to be unstable. Figure 3(d) is a graph showing the evolution in time from initial values of the interface $d$.

Figure 3(e) is a graph showing $0.6[\mathrm{~m}]$ contour lines obtained after 380 hours, starting from initial values for the interface $d$ and the water level $\zeta$ under Cond. 4. For the case with a density difference, it is found with both the water level $\zeta$ and the interface $d$ that the contour lines are perpendicular to the wind direction. Again, in this case, the values for the water level $\zeta$ are higher under the wind, whereas the values for the interface $d$ are lower under the wind. Figure 3(f) is a graph based on computations under Cond. 4, showing the evolution in time of the interface $d$ and the water level $\zeta$ starting from initial values. It is found that the values converge in both $d$ and

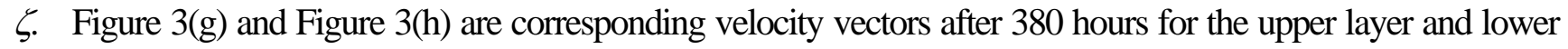
layer, respectively. It is noted that relatively high speed appears near on $\Gamma_{2}$. This may come from the boundary conditions on $\Gamma_{2}$. Some improvement may be required.

It is also found for the present problem that the interface is unstable in the absence of a density difference, whereas results indicating the same stable interface as with Mikawa Bay are obtained when a density difference is applied. The density difference is a little larger. However, more smaller density difference could not give a stable solution. This $10 \%$ difference seems to be a limited density difference and it is concluded that such a big density difference can only produce this type of two layer flows under the present conditions. Furthermore, it is conjectured that the interface is always unstable in the absence of the density difference. So, the one-layer model should be used in that case. Authors further believe that the two-layer model should not be used in that case because it necessarily produces numerical instability which comes from the instability of the interface (the interfacial instability [9]) and not from the instability of our finite element scheme. The similar situation may happen if the density difference is not so sufficient and it is a little difficult to compute such a two-layer flow. It is strongly hoped that these conjectures will be rigorously justified in future.

Fig. 3. (a) Contour map of $\zeta$ after 55 hours (Cond. 3)

(b) Transmition of $\zeta$ (Cond. 3) 
(c) Contour map of $\left(d+H_{0}^{u}\right)$ after 55 hours (Cond. 3)

(d) Transmition of $\left(d+H_{0}^{u}\right)$ (Cond. 3)

(e) Contour map of $\zeta$ and $\left(d+H_{0}^{u}\right)$ after 380 hours (Cond. 4)

(f) Transmition of $\zeta$ and $\left(d+H_{0}^{u}\right)$ (Cond. 4)

(g) Velocity vectors of upper layer after 380 hours (Cond. 4)

(h) Velocity vectors of lower layer after 380 hours (Cond. 4)

\section{Concluding remarks}

In this study, a numerical scheme for the solution of shallow-water equations using the layer model based on the method in [4] has been presented. When densities are the same in the derived two-layer viscous shallow-water equations, it is found that the equations for the upper layer and lower layer are combined to produce a form equivalent to the one-layer equations. Moreover, it is numerically demonstrated that interfacial instability produced when densities are the same can be resolved by providing a suitable density difference.

Since observed data are not got easily, we show results of a simple physical experiment, which demonstrate that stratification of the two layer model really happens if a sufficient condition is given. Figure 4 shows a two layer flow produced by a simple physical experiment. Three driers blow from the right hand, yielding an upper oil layer which is higher under the wind and a lower water layer which is lower under the wind.

Acknowledgements. Kanayama (1981) [2] and others have carried out analyses regarding Mikawa Bay, and Kyozuka, Furuse and Kimura (2002) [8] and Aoyama, Ikeda and Takatori [1] have carried out analyses regarding the Ariake Sea. We would like to make specific mention that these investigations were referenced regarding data 
collection in ocean regions such as wind speed [1] and physical values [2]. Finally, authors would like to mention sincere thanks to referees of this paper for their crucial advices, without which this paper would not have appeared for ever.

\section{REFERENCES}

[1] T. Aoyama, K. Ikeda and A. Takatori, Study on Closing Water Area, Environmental Research Institute Tokyo home page, http://eritokyo.jp/independent/gulf/index.html (in Japanese)

[2] H. Kanayama, A numerical experiment on the wind-driven flow in Mikawa Bay, Proceedings of 15th Symposium on Matrix Analysis Method, JSSC, (1981), pp.407-412. (in Japanese)

[3] H. Kanayama and T. Ishikawa, A finite element scheme of the two layer viscous shallow water equations, Proceedings of 16th Computational Fluid Dynamics Symposium, D29-1, (2002). (in Japanese)

[4] H. Kanayama and K. Ohtsuka, Finite element analysis on the tidal current and COD distribution in Mikawa Bay, Coastal Engineering in Japan, Vol.21, (1978), pp. 157-171.

[5] H. Kanayama and T. Ushijima, On the viscous shallow-water equations I -- Derivation and conservation laws --, Memoirs of Numerical Mathematics 8/9, (1981/1982), pp. 39-64.

[6] H. Kanayama and T. Ushijima, On the viscous shallow-water equations II --A linearized system--, Bulletin of University of Electro-Communications, Vol.1, No.2, (1988), pp.347-355.

[7] H. Kanayama and T. Ushijima, On the viscous shallow-water equations III --A finite element scheme-- , Bulletin of University of Electro-Communications, Vol.2, No.1, (1989), pp.47-62.

[8] Y. Kyozuka, K. Furusho and Y. Kimura, A numerical simulation of water flow by opening the floodgate of embankment in Isahaya Bay, Engineering Sciences Reports, Kyushu University, Vol.23, No.2, (2002), pp.207-215. (in Japanese)

[9] K. Murakami, Calculations of vertical circulation in stratified waters by 2-level and 2-layer models, Proceedings of Coastal Engineering, JSCE, Vol.36, (1989), pp. 204-208. (in Japanese) 
[10] T. Sawaragi(ed.) : New Coastal Engineering in Global Environment, (Fuji Technosystem Co., 2002), pp.89-96. (in Japanese)

[11] M. Tabata, A finite element approximation corresponding to the upwind finite differencing, Memoirs of Numerical Mathematics, Vol.4, (1977), pp. 47-63. 


\section{Appendix 1}

The integration of (2-1) from $b$ to $d$ in the $x_{3}$ direction yields

$$
\sum_{j=1}^{3} \int_{b}^{d} \frac{\partial}{\partial x_{j}} u_{j} d x_{3}=0
$$

In the above,

$$
\int_{b}^{d} \frac{\partial}{\partial x_{3}} u_{3} d x_{3}=\left.u_{3}\right|_{x_{3}=b} ^{x_{3}=d}
$$

Moreover, for $i=1$ and $i=2$,

$$
\int_{b}^{d} \frac{\partial}{\partial x_{i}} u_{i} d x_{3}=\frac{\partial}{\partial x_{i}} \int_{b}^{d} u_{i} d x_{3}-\left.\left(u_{i} \frac{\partial}{\partial x_{i}} x_{3}\right)\right|_{x_{3}=b} ^{x_{3}=d}
$$

Hence,

$$
\sum_{j=1}^{2} \frac{\partial}{\partial x_{j}} \int_{b}^{d} u_{j} d x_{3}+\left.\left(u_{3}-\sum_{j=1}^{2} u_{j} \frac{\partial}{\partial x_{j}} x_{3}\right)\right|_{x_{3}=b} ^{x_{3}=d}=0
$$

From (2-5), it holds that

$$
\left.\left(u_{3}-\sum_{j=1}^{2} u_{j} \frac{\partial}{\partial x_{j}} x_{3}\right)\right|_{x_{3}=b} ^{x_{3}=d}=\frac{\partial}{\partial t} d
$$

Thus, we have

$$
\frac{\partial}{\partial t} d+\sum_{j=1}^{2} \frac{\partial}{\partial x_{j}}\left(H^{l} U_{j}^{l}\right)=0
$$

Similarly, the integration of $(2-1)$ from $d$ to $\zeta$ in the $x_{3}$ direction yields

$$
\frac{\partial}{\partial t}(\zeta-d)+\sum_{j=1}^{2} \frac{\partial}{\partial x_{j}}\left(H^{u} U_{j}^{u}\right)=0
$$

Now, we integrate (2-4) in the $x_{3}$ direction. In the case of the upper layer $\left(x_{3}=d \sim \zeta\right.$ ),

$$
0=-p-\rho^{u} g x_{3}+C_{1}
$$


where $C_{1}$ is a constant. Since the atmospheric pressure $p_{\text {air }}$ is assumed to be constant on the sea surface, it holds that

$$
p_{\text {air }}=-\rho^{u} g \zeta+C_{1} .
$$

Therefore,

$$
p=-\rho^{u} g x_{3}+\rho^{u} g \zeta+p_{a i r} .
$$

In the case of the lower layer $\left(x_{3}=b \sim d\right)$,

$$
0=-p-\rho^{l} g x_{3}+C_{2},
$$

where $C_{2}$ is another constant. Since $p$ should be continuous at the interface $\left(x_{3}=d\right)$, it holds that

$$
\rho^{u} g(\zeta-d)+p_{\text {air }}=-\rho^{l} g d+C_{2} .
$$

Therefore,

$$
p=-\rho^{l} g x_{3}+\rho^{u} g \zeta+\left(\rho^{l}-\rho^{u}\right) g d+p_{\text {air }} .
$$

The integration of (2-2) from $b$ to $d$ in the $x_{3}$ direction yields

$$
\int_{b}^{d} \frac{\partial}{\partial t} u_{1} d x_{3}+\sum_{j=1}^{3} \int_{b}^{d} u_{j} \frac{\partial}{\partial x_{j}} u_{1} d x_{3}=-\int_{b}^{d} \frac{1}{\rho^{l}} \frac{\partial p}{\partial x_{1}} d x_{3}+\frac{1}{\rho^{l}} \sum_{j=1}^{3} \int_{b}^{d} \frac{\partial}{\partial x_{j}} \tau_{1 j} d x_{3}+\int_{b}^{d} f u_{2} d x_{3} .
$$

Then,

$$
\begin{aligned}
& \int_{b}^{d} \frac{\partial}{\partial t} u_{1} d x_{3}+\sum_{j=1}^{3} \int_{b}^{d} u_{j} \frac{\partial}{\partial x_{j}} u_{1} d x_{3} \\
& =\int_{b}^{d} \frac{\partial}{\partial t} u_{1} d x_{3}+\sum_{j=1}^{3} \int_{b}^{d}\left(\frac{\partial}{\partial x_{j}}\left(u_{j} u_{1}\right)-u_{1} \frac{\partial}{\partial x_{j}} u_{j}\right) d x_{3}
\end{aligned}
$$

From (2-1), $\sum_{j=1}^{3} \int_{b}^{d} u_{1} \frac{\partial}{\partial x_{j}} u_{j} d x_{3}=\int_{b}^{d} u_{1} \sum_{j=1}^{3} \frac{\partial}{\partial x_{j}} u_{j} d x_{3}=0$. 


$$
\begin{aligned}
& =\frac{\partial}{\partial t} \int_{b}^{d} u_{1} d x_{3}+\sum_{j=1}^{2} \frac{\partial}{\partial x_{j}} \int_{b}^{d} u_{j} u_{1} d x_{3}+\left.\left\{u_{1}\left(u_{3}-\frac{\partial}{\partial t} x_{3}-\sum_{j=1}^{2} u_{j} \frac{\partial}{\partial x_{j}} x_{3}\right)\right\}\right|_{x_{3}=b} ^{x_{3}=d} \\
& \left(\text { From (2-5), }\left.\left\{u_{1}\left(u_{3}-\frac{\partial}{\partial t} x_{3}-\sum_{j=1}^{2} u_{j} \frac{\partial}{\partial x_{j}} x_{3}\right)\right\}\right|_{x_{3}=b} ^{x_{3}=d}=0 .\right. \\
& =\frac{\partial}{\partial t} \int_{b}^{d} u_{1} d x_{3}+\sum_{j=1}^{2} \frac{\partial}{\partial x_{j}} \int_{b}^{d} u_{j} u_{1} d x_{3} \\
& =\frac{\partial}{\partial t} \int_{b}^{d} u_{1} d x_{3}+\frac{\partial}{\partial x_{1}} \int_{b}^{d}\left(\left(u_{1}\right)^{2}-2 u_{1} U_{1}^{l}+\left(U_{1}^{l}\right)^{2}\right) d x_{3}+\frac{\partial}{\partial x_{2}} \int_{b}^{d}\left(u_{1} u_{2}-u_{1} U_{2}^{l}-u_{2} U_{1}^{l}+U_{1}^{l} U_{2}^{l}\right) d x_{3} \\
& +\frac{\partial}{\partial x_{1}} \int_{b}^{d}\left(2 u_{1} U_{1}^{l}-\left(U_{1}^{l}\right)^{2}\right) d x_{3}+\frac{\partial}{\partial x_{2}} \int_{b}^{d}\left(u_{1} U_{2}^{l}+U_{1}^{l} u_{2}-U_{1}^{l} U_{2}^{l}\right) d x_{3} \\
& =\frac{\partial}{\partial t}\left(H^{l} U_{1}^{l}\right)+\frac{\partial}{\partial x_{1}}\left\{H^{l}\left(U_{1}^{l}\right)^{2}\right\}+\frac{\partial}{\partial x_{2}}\left(H^{l} U_{1}^{l} U_{2}^{l}\right)+\frac{\partial}{\partial x_{1}} \int_{b}^{d}\left(u_{1}-U_{1}^{l}\right)^{2} d x_{3} \\
& +\frac{\partial}{\partial x_{2}} \int_{b}^{d}\left(u_{1}-U_{1}^{l}\right)\left(u_{2}-U_{2}^{l}\right) d x_{3}
\end{aligned}
$$

(Set $\quad \tilde{u}_{1}^{l}=u_{1}-U_{1}^{l} \quad$ and $\quad \tilde{u}_{2}^{l}=u_{2}-U_{2}^{l}$. )$$
=\frac{\partial}{\partial t}\left(H^{l} U_{1}^{l}\right)+\frac{\partial}{\partial x_{1}}\left\{H^{l}\left(U_{1}^{l}\right)^{2}\right\}+\frac{\partial}{\partial x_{2}}\left(H^{l} U_{1}^{l} U_{2}^{l}\right)+\frac{\partial}{\partial x_{1}} \int_{b}^{d}\left(\tilde{u}_{1}^{l}\right)^{2} d x_{3}+\frac{\partial}{\partial x_{2}} \int_{b}^{d} \tilde{u}_{1}^{l} \tilde{u}_{2}^{l} d x_{3} .
$$

Here, $\frac{\partial}{\partial x_{1}} \int_{b}^{d}\left(\tilde{u}_{1}^{l}\right)^{2} d x_{3}+\frac{\partial}{\partial x_{2}} \int_{b}^{d} \tilde{u}_{1}^{l} \tilde{u}_{2}^{l} d x_{3}$ is sent to the right hand side. Then,

$$
\begin{aligned}
& \frac{\partial}{\partial t}\left(H^{l} U_{1}^{l}\right)+\frac{\partial}{\partial x_{1}}\left\{H^{l}\left(U_{1}^{l}\right)^{2}\right\}+\frac{\partial}{\partial x_{2}}\left(H^{l} U_{1}^{l} U_{2}^{l}\right) \\
& =-\int_{b}^{d} \frac{1}{\rho^{l}} \frac{\partial p}{\partial x_{1}} d x_{3}+\frac{1}{\rho^{l}} \sum_{j=1}^{3} \int_{b}^{d} \frac{\partial}{\partial x_{j}} \tau_{1 j} d x_{3}-\frac{\partial}{\partial x_{1}} \int_{b}^{d}\left(\tilde{u}_{1}^{l}\right)^{2} d x_{3}-\frac{\partial}{\partial x_{2}} \int_{b}^{d} \tilde{u}_{1}^{l} \tilde{u}_{2}^{l} d x_{3}+\int_{b}^{d} f u_{2} d x_{3} .
\end{aligned}
$$

On the other hand, we have

$$
\begin{aligned}
& \frac{\partial}{\partial t}\left(H^{l} U_{1}^{l}\right)+\frac{\partial}{\partial x_{1}}\left\{H^{l}\left(U_{1}^{l}\right)^{2}\right\}+\frac{\partial}{\partial x_{2}}\left(H^{l} U_{1}^{l} U_{2}^{l}\right) \\
& =H^{l} \frac{\partial}{\partial t} U_{1}^{l}+U_{1}^{l} \frac{\partial}{\partial t} H^{l}+U_{1}^{l} \frac{\partial}{\partial x_{1}}\left(H^{l} U_{1}^{l}\right)+H^{l} U_{l}^{l} \frac{\partial}{\partial x_{1}} U_{1}^{l}+U_{1}^{l} \frac{\partial}{\partial x_{2}}\left(H^{l} U_{2}^{l}\right)+H^{l} U_{2}^{l} \frac{\partial}{\partial x_{2}} U_{1}^{l}
\end{aligned}
$$




$$
\begin{aligned}
= & H^{l} \frac{\partial}{\partial t} U_{1}^{l}+H^{l} U_{1}^{l} \frac{\partial}{\partial x_{1}} U_{1}^{l}+H^{l} U_{2}^{l} \frac{\partial}{\partial x_{2}} U_{1}^{l}+U_{1}^{l}\left(\frac{\partial}{\partial t}(d-b)+\frac{\partial}{\partial x_{1}}\left(H^{l} U_{1}^{l}\right)+\frac{\partial}{\partial x_{2}}\left(H^{l} U_{2}^{l}\right)\right) \\
& \left(\text { From }(2-6 l), U_{1}^{l}\left(\frac{\partial}{\partial t}(d-b)+\frac{\partial}{\partial x_{1}}\left(H^{l} U_{1}^{l}\right)+\frac{\partial}{\partial x_{2}}\left(H^{l} U_{2}^{l}\right)\right)=0 .\right) \\
= & H^{l}\left(\frac{\partial}{\partial t} U_{1}^{l}+U_{1}^{l} \frac{\partial}{\partial x_{1}} U_{1}^{l}+U_{2}^{l} \frac{\partial}{\partial x_{2}} U_{1}^{l}\right) .
\end{aligned}
$$

Now, we change the right hand side of $(2-7 a)$. For the pressure term, since we have, by $(2-8 l)$,

$$
\frac{\partial p}{\partial x_{1}}=\rho^{u} g \frac{\partial \zeta}{\partial x_{1}}+\left(\rho^{l}-\rho^{u}\right) g \frac{\partial d}{\partial x_{1}}
$$

it holds that

$$
\begin{aligned}
& -\int_{b}^{d} \frac{1}{\rho^{l}} \frac{\partial p}{\partial x_{1}} d x_{3}=-\int_{b}^{d}\left(g \frac{\rho^{u}}{\rho^{l}} \frac{\partial \zeta}{\partial x_{1}}+g \frac{\rho^{l}-\rho^{u}}{\rho^{l}} \frac{\partial d}{\partial x_{1}}\right) d x_{3} \\
& =-g H^{l}\left(\frac{\rho^{u}}{\rho^{l}} \frac{\partial \zeta}{\partial x_{1}}+\frac{\rho^{l}-\rho^{u}}{\rho^{l}} \frac{\partial d}{\partial x_{1}}\right) .
\end{aligned}
$$

For the stress terms, it holds that

$$
\begin{aligned}
& \frac{1}{\rho^{l}} \sum_{j=1}^{3} \int_{b}^{d} \frac{\partial}{\partial x_{j}} \tau_{1 j} d x_{3}-\frac{\partial}{\partial x_{1}} \int_{b}^{d}\left(\tilde{u}_{1}^{l}\right)^{2} d x_{3}-\frac{\partial}{\partial x_{2}} \int_{b}^{d} \tilde{u}_{1}^{l} \tilde{u}_{2}^{l} d x_{3} \\
& =\frac{1}{\rho^{l}} \frac{\partial}{\partial x_{1}} \int_{b}^{d} \tau_{11} d x_{3}-\frac{\partial}{\partial x_{1}} \int_{b}^{d}\left(\tilde{u}_{1}^{l}\right)^{2} d x_{3}+\frac{1}{\rho^{l}} \frac{\partial}{\partial x_{2}} \int_{b}^{d} \tau_{12} d x_{3} \\
& -\frac{\partial}{\partial x_{2}} \int_{b}^{d} \tilde{u}_{1}^{l} \tilde{u}_{2}^{l} d x_{3}+\left.\frac{1}{\rho^{l}}\left(\tau_{13}-\tau_{11} \frac{\partial}{\partial x_{1}} x_{3}-\tau_{12} \frac{\partial}{\partial x_{2}} x_{3}\right)\right|_{x_{3}=b} ^{x_{3}=d}
\end{aligned}
$$

Here, set

$$
\frac{1}{\rho^{l}}\left(S_{1}^{l}-B_{1}^{l}\right)=\left.\frac{1}{\rho^{l}}\left(\tau_{13}-\tau_{11} \frac{\partial}{\partial x_{1}} x_{3}-\tau_{12} \frac{\partial}{\partial x_{2}} x_{3}\right)\right|_{x_{3}=b} ^{x_{3}=d}
$$

We have the following experimental expressions for $S_{1}^{l}$ and $B_{1}^{l}$,

$$
\frac{1}{\rho^{l}} S_{1}^{l}=\frac{2 \mu\left(U_{1}^{u}-U_{1}^{l}\right)}{\rho^{l}(\zeta-b)} \text { and }
$$




$$
\frac{1}{\rho^{l}} B_{1}^{l}=\frac{g}{C^{2}} U_{1}\left(\left(U_{1}\right)^{2}+\left(U_{2}\right)^{2}\right)^{\frac{1}{2}}
$$

$$
\text { where } \quad U_{i}=\frac{H^{u} U_{i}^{u}+H^{l} U_{i}^{l}}{H^{u}+H^{l}} \text {. }
$$

Moreover, set

$$
\begin{aligned}
& \tilde{\tau}_{11}^{l}=\frac{1}{H^{l}} \int_{b}^{d} \tau_{11} d x_{3}-\frac{\rho^{l}}{H^{l}} \int_{b}^{d}\left(\tilde{u}_{1}^{l}\right)^{2} d x_{3}, \\
& \tilde{\tau}_{12}^{l}=\frac{1}{H^{l}} \int_{b}^{d} \tau_{12} d x_{3}-\frac{\rho^{l}}{H^{l}} \int_{b}^{d} \tilde{u}_{1}^{l} \tilde{u}_{2}^{l} d x_{3} .
\end{aligned}
$$

Then, we have

$$
\begin{aligned}
& -\int_{b}^{d} \frac{1}{\rho^{l}} \frac{\partial p}{\partial x_{1}} d x_{3}+\frac{1}{\rho^{l}} \sum_{j=1}^{3} \int_{b}^{d} \frac{\partial}{\partial x_{j}} \tau_{1 j} d x_{3}-\frac{\partial}{\partial x_{1}} \int_{b}^{d}\left(\tilde{u}_{1}^{l}\right)^{2} d x_{3}-\frac{\partial}{\partial x_{2}} \int_{b}^{d} \tilde{u}_{1}^{l} \tilde{u}_{2}^{l} d x_{3}+\int_{b}^{d} f u_{2} d x_{3} \\
& =-g H^{l}\left(\frac{\rho^{u}}{\rho^{l}} \frac{\partial \zeta}{\partial x_{1}}+\frac{\rho^{l}-\rho^{u}}{\rho^{l}} \frac{\partial d}{\partial x_{1}}\right)+\frac{1}{\rho^{l}}\left(\frac{\partial}{\partial x_{1}}\left(H^{l} \tilde{\tau}_{11}^{l}\right)+\frac{\partial}{\partial x_{2}}\left(H^{l} \tilde{\tau}_{12}^{l}\right)\right) \\
& +\frac{2 \mu\left(U_{1}^{u}-U_{1}^{l}\right)}{\rho^{l}(\zeta-b)}-\frac{g}{C^{2}} U_{1}\left(\left(U_{1}\right)^{2}+\left(U_{2}\right)^{2}\right)^{\frac{1}{2}}+f H^{l} U_{2}^{l} .
\end{aligned}
$$

Therefore, for the lower layer, $(2-7 a)$ becomes

$$
\begin{aligned}
& H^{l}\left(\frac{\partial}{\partial t} U_{1}^{l}+U_{1}^{l} \frac{\partial}{\partial x_{1}} U_{1}^{l}+U_{2}^{l} \frac{\partial}{\partial x_{2}} U_{1}^{l}\right) \\
& =-g H^{l}\left(\frac{\rho^{u}}{\rho^{l}} \frac{\partial \zeta}{\partial x_{1}}+\frac{\rho^{l}-\rho^{u}}{\rho^{l}} \frac{\partial d}{\partial x_{1}}\right)+\frac{1}{\rho^{l}}\left(\frac{\partial}{\partial x_{1}}\left(H^{l} \widetilde{\tau}_{11}^{l}\right)+\frac{\partial}{\partial x_{2}}\left(H^{l} \widetilde{\tau}_{12}^{l}\right)\right) \\
& +\frac{2 \mu\left(U_{1}^{u}-U_{1}^{l}\right)}{\rho^{l}(\zeta-b)}-\frac{g}{C^{2}} U_{1}\left(\left(U_{1}\right)^{2}+\left(U_{2}\right)^{2}\right)^{\frac{1}{2}}+f H^{l} U_{2}^{l} .
\end{aligned}
$$

Similarly, the integration of (2-2) from $d$ to $\zeta$ in the $x_{3}$ direction yields

$$
\begin{aligned}
\int_{d}^{\zeta} \frac{\partial}{\partial t} u_{1} d x_{3}+\sum_{j=1}^{3} \int_{d}^{\zeta} u_{j} \frac{\partial}{\partial x_{j}} u_{1} d x_{3} \\
=-\int_{d}^{\zeta} \frac{1}{\rho^{u}} \frac{\partial p}{\partial x_{1}} d x_{3}+\frac{1}{\rho^{u}} \sum_{j=1}^{3} \int_{d}^{\zeta} \frac{\partial}{\partial x_{j}} \tau_{1 j} d x_{3}+\int_{d}^{\zeta} f u_{2} d x_{3} .
\end{aligned}
$$


Then,

$$
\begin{aligned}
& \int_{d}^{\zeta} \frac{\partial}{\partial t} u_{1} d x_{3}+\sum_{j=1}^{3} \int_{d}^{\zeta} u_{j} \frac{\partial}{\partial x_{j}} u_{1} d x_{3} \\
& =\frac{\partial}{\partial t}\left(H^{u} U_{1}^{u}\right)+\frac{\partial}{\partial x_{1}}\left\{H^{u}\left(U_{1}^{u}\right)^{2}\right\}+\frac{\partial}{\partial x_{2}}\left(H^{u} U_{1}^{u} U_{2}^{u}\right) \\
& +\frac{\partial}{\partial x_{1}} \int_{d}^{\zeta}\left(u_{1}-U_{1}^{u}\right)^{2} d x_{3}+\frac{\partial}{\partial x_{2}} \int_{d}^{\zeta}\left(u_{1}-U_{1}^{u}\right)\left(u_{2}-U_{2}^{u}\right) d x_{3} \\
& \left(\text { Set } \quad \tilde{u}_{1}^{u}=u_{1}-U_{1}^{u} \text { and } \quad \tilde{u}_{2}^{u}=u_{2}-U_{2}^{u} \cdot\right] \\
& =\frac{\partial}{\partial t}\left(H^{u} U_{1}^{u}\right)+\frac{\partial}{\partial x_{1}}\left\{H^{u}\left(U_{1}^{u}\right)^{2}\right\}+\frac{\partial}{\partial x_{2}}\left(H^{u} U_{1}^{u} U_{2}^{u}\right)+\frac{\partial}{\partial x_{1}} \int_{d}^{\zeta}\left(\tilde{u}_{1}^{u}\right)^{2} d x_{3}+\frac{\partial}{\partial x_{2}} \int_{d}^{\zeta} \tilde{u}_{1}^{u} \tilde{u}_{2}^{u} d x_{3} .
\end{aligned}
$$

Here, $\frac{\partial}{\partial x_{1}} \int_{d}^{\zeta}\left(\tilde{u}_{1}^{u}\right)^{2} d x_{3}+\frac{\partial}{\partial x_{2}} \int_{d}^{\zeta} \tilde{u}_{1}^{u} \tilde{u}_{2}^{u} d x_{3}$ is sent to the right hand side. Then,

$$
\begin{aligned}
& \frac{\partial}{\partial t}\left(H^{u} U_{1}^{u}\right)+\frac{\partial}{\partial x_{1}}\left\{H^{u}\left(U_{1}^{u}\right)^{2}\right\}+\frac{\partial}{\partial x_{2}}\left(H^{u} U_{1}^{u} U_{2}^{u}\right) \\
& =-\int_{d}^{\zeta} \frac{1}{\rho^{u}} \frac{\partial p}{\partial x_{1}} d x_{3}+\frac{1}{\rho^{u}} \sum_{j=1}^{3} \int_{d}^{\zeta} \frac{\partial}{\partial x_{j}} \tau_{1 j} d x_{3} \\
& -\frac{\partial}{\partial x_{1}} \int_{d}^{\zeta}\left(\tilde{u}_{1}^{u}\right)^{2} d x_{3}-\frac{\partial}{\partial x_{2}} \int_{d}^{\zeta} \tilde{u}_{1}^{u} \tilde{u}_{2}^{u} d x_{3}+\int_{d}^{\zeta} f u_{2} d x_{3} .
\end{aligned}
$$

Again, we have

$$
\begin{array}{r}
\frac{\partial}{\partial t}\left(H^{u} U_{1}^{u}\right)+\frac{\partial}{\partial x_{1}}\left\{H^{u}\left(U_{1}^{u}\right)^{2}\right\}+\frac{\partial}{\partial x_{2}}\left(H^{u} U_{1}^{u} U_{2}^{u}\right) \\
=H^{u}\left(\frac{\partial}{\partial t} U_{1}^{u}+U_{1}^{u} \frac{\partial}{\partial x_{1}} U_{1}^{u}+U_{2}^{u} \frac{\partial}{\partial x_{2}} U_{1}^{u}\right)
\end{array}
$$

Also, we change the right hand side of $(2-7 b)$. For the pressure term, we have, from $(2-8 u)$,

$$
-\int_{d}^{\zeta} \frac{1}{\rho^{u}} \frac{\partial p}{\partial x_{1}} d x_{3}=-g H^{u} \frac{\partial \zeta}{\partial x_{1}} .
$$


For the stress terms, since we have the following experimental expressions for $S_{1}^{l}$ and $B_{1}^{l}$,

$$
\frac{1}{\rho^{u}} S_{1}^{u}=\frac{\theta \rho_{a}}{\rho^{u}} W_{1}\left(\left(W_{1}\right)^{2}+\left(W_{2}\right)^{2}\right)^{\frac{1}{2}}
$$

and

$$
\frac{1}{\rho^{u}} B_{1}^{u}=\frac{2 \mu\left(U_{1}^{u}-U_{1}^{l}\right)}{\rho^{u}(\zeta-b)},
$$

with

$$
\tilde{\tau}_{11}^{u}=\frac{1}{H^{u}} \int_{d}^{\zeta} \tau_{11} d x_{3}-\frac{\rho^{u}}{H^{u}} \int_{d}^{\zeta}\left(\tilde{u}_{1}^{u}\right)^{2} d x_{3},
$$

and

$$
\tilde{\tau}_{12}^{u}=\frac{1}{H^{u}} \int_{d}^{\zeta} \tau_{12} d x_{3}-\frac{\rho^{u}}{H^{u}} \int_{d}^{\zeta} \tilde{u}_{1}^{u} \tilde{u}_{2}^{u} d x_{3},
$$

it holds that

$$
\begin{aligned}
& -\int_{d}^{\zeta} \frac{1}{\rho^{u}} \frac{\partial p}{\partial x_{1}} d x_{3}+\frac{1}{\rho^{u}} \sum_{j=1}^{3} \int_{d}^{\zeta} \frac{\partial}{\partial x_{j}} \tau_{1 j} d x_{3}-\frac{\partial}{\partial x_{1}} \int_{d}^{\zeta}\left(\tilde{u}_{1}^{u}\right)^{2} d x_{3}-\frac{\partial}{\partial x_{2}} \int_{d}^{\zeta} \tilde{u}_{1}^{u} \tilde{u}_{2}^{u} d x_{3}+\int_{d}^{\zeta} f u_{2} d x_{3} \\
& =-g H^{u}\left(\frac{\partial \zeta}{\partial x_{1}}\right)+\frac{1}{\rho^{u}}\left(\frac{\partial}{\partial x_{1}}\left(H^{u} \tilde{\tau}_{11}^{u}\right)+\frac{\partial}{\partial x_{2}}\left(H^{u} \tilde{\tau}_{12}^{u}\right)\right) \\
& +\frac{\theta \rho_{a}}{\rho^{u}} W_{1}\left(\left(W_{1}\right)^{2}+\left(W_{2}\right)^{2}\right)^{\frac{1}{2}}-\frac{2 \mu\left(U_{1}^{u}-U_{1}^{l}\right)}{\rho^{u}(\zeta-b)}+f H^{u} U_{2}^{u} .
\end{aligned}
$$

Therefore, for the upper layer, $(2-7 b)$ becomes

$$
\begin{aligned}
& H^{u}\left(\frac{\partial}{\partial t} U_{1}^{u}+U_{1}^{u} \frac{\partial}{\partial x_{1}} U_{1}^{u}+U_{2}^{u} \frac{\partial}{\partial x_{2}} U_{1}^{u}\right) \\
& =-g H^{u}\left(\frac{\partial \zeta}{\partial x_{1}}\right)+\frac{1}{\rho^{u}}\left(\frac{\partial}{\partial x_{1}}\left(H^{u} \tilde{\tau}_{11}^{u}\right)+\frac{\partial}{\partial x_{2}}\left(H^{u} \tilde{\tau}_{12}^{u}\right)\right)+\frac{\theta \rho_{a}}{\rho^{u}} W_{1}\left(\left(W_{1}\right)^{2}+\left(W_{2}\right)^{2}\right)^{\frac{1}{2}}-\frac{2 \mu\left(U_{1}^{u}-U_{1}^{l}\right)}{\rho^{u}(\zeta-b)}+f H^{u} U_{2}^{u} .
\end{aligned}
$$

The integration of $(2-3)$ is omitted. 
Appendix 2

Addition is performed separately. The addition of the pressure terms yields

$$
-g H^{u} \frac{\partial \zeta}{\partial x_{i}}-g H^{l}\left(\frac{\rho^{u}}{\rho^{l}} \frac{\partial \zeta}{\partial x_{i}}+\frac{\rho^{l}-\rho^{u}}{\rho^{l}} \frac{\partial d}{\partial x_{i}}\right)=-g H \frac{\partial \zeta}{\partial x_{i}} .
$$

Addition of friction terms also yields

$$
\begin{aligned}
& \frac{\theta \rho_{a}}{\rho} W_{i}\left(\left(W_{1}\right)^{2}+\left(W_{2}\right)^{2}\right)^{\frac{1}{2}}-\frac{2 \mu\left(U_{i}^{u}-U_{i}^{l}\right)}{\rho(\zeta-b)} \\
& +\frac{2 \mu\left(U_{i}^{u}-U_{i}^{l}\right)}{\rho(\zeta-b)}-\frac{g}{C^{2}} U_{i}\left(\left(U_{1}\right)^{2}+\left(U_{2}\right)^{2}\right)^{\frac{1}{2}} \\
& =\frac{\theta \rho_{a}}{\rho} W_{i}\left(\left(W_{1}\right)^{2}+\left(W_{2}\right)^{2}\right)^{\frac{1}{2}}-\frac{g}{C^{2}} U_{i}\left(\left(U_{1}\right)^{2}+\left(U_{2}\right)^{2}\right)^{\frac{1}{2}}
\end{aligned}
$$

The Coriolis force terms produce

$$
f H^{u} U_{i+1}^{u}+f H^{l} U_{i+1}^{l}=f\left(\int_{d}^{\zeta} u_{i+1} d x_{3}+\int_{b}^{d} u_{i+1} d x_{3}\right)=f H U_{i+1} .
$$

Finally addition of other terms yields

$$
\begin{aligned}
& H^{u} \frac{\partial}{\partial t} U_{i}^{u}+\sum_{j=1}^{2} H^{u} U_{j}^{u} \frac{\partial}{\partial x_{j}} U_{i}^{u}-\frac{1}{\rho^{u}}\left(\sum_{j=1}^{2} \frac{\partial}{\partial x_{j}} H^{u} \tilde{\tau}_{i j}^{u}\right) \\
& +H^{l} \frac{\partial}{\partial t} U_{i}^{l}+\sum_{j=1}^{2} H^{l} U_{j}^{l} \frac{\partial}{\partial x_{j}} U_{i}^{l}-\frac{1}{\rho^{l}}\left(\sum_{j=1}^{2} \frac{\partial}{\partial x_{j}} H^{l} \tilde{\tau}_{i j}^{l}\right) \\
& =\frac{\partial}{\partial t}\left(H^{u} U_{i}^{u}\right)+\sum_{j=1}^{2} \frac{\partial}{\partial x_{j}}\left(H^{u} U_{j}^{u} U_{i}^{u}\right)-\frac{1}{\rho^{u}}\left(\sum_{j=1}^{2} \frac{\partial}{\partial x_{j}} H^{u} \tilde{\tau}_{i j}^{u}\right) \\
& +\frac{\partial}{\partial t}\left(H^{l} U_{i}^{l}\right)+\sum_{j=1}^{2} \frac{\partial}{\partial x_{j}}\left(H^{l} U_{j}^{l} U_{i}^{l}\right)-\frac{1}{\rho^{l}}\left(\sum_{j=1}^{2} \frac{\partial}{\partial x_{j}} H^{l} \tilde{\tau}_{i j}^{l}\right) \\
& =\frac{\partial}{\partial t}\left(H^{u} U_{i}^{u}\right)+\sum_{j=1}^{2} \frac{\partial}{\partial x_{j}}\left(H^{u} U_{j}^{u} U_{i}^{u}\right)-\frac{1}{\rho^{u}}\left(\sum_{j=1}^{2} \frac{\partial}{\partial x_{j}}\left(\int_{d}^{\zeta} \tau_{i j} d x_{3}-\rho^{u} \int_{d}^{\zeta} \tilde{u}_{i}^{u} \tilde{u}_{j}^{u} d x_{3}\right)\right)
\end{aligned}
$$




$$
\begin{aligned}
& +\frac{\partial}{\partial t}\left(H^{l} U_{i}^{l}\right)+\sum_{j=1}^{2} \frac{\partial}{\partial x_{j}}\left(H^{l} U_{j}^{l} U_{i}^{l}\right)-\frac{1}{\rho^{l}}\left(\sum_{j=1}^{2} \frac{\partial}{\partial x_{j}}\left(\int_{b}^{d} \tau_{i j} d x_{3}-\rho^{l} \int_{b}^{d} \tilde{u}_{i}^{l} \tilde{u}_{j}^{l} d x_{3}\right)\right) \\
& =\frac{\partial}{\partial t}\left(H U_{i}\right)-\frac{1}{\rho}\left(\sum_{j=1}^{2} \frac{\partial}{\partial x_{j}} \int_{b}^{\zeta} \tau_{i j} d x_{3}\right) \\
& +\sum_{j=1}^{2} \frac{\partial}{\partial x_{j}}\left(H^{u} U_{j}^{u} U_{i}^{u}+H^{l} U_{j}^{l} U_{i}^{l}+\int_{d}^{\zeta} \tilde{u}_{i}^{u} \tilde{u}_{j}^{u} d x_{3}+\int_{b}^{d} \tilde{u}_{i}^{l} \tilde{u}_{j}^{l} d x_{3}\right) \\
& =\frac{\partial}{\partial t}\left(H U_{i}\right)-\frac{1}{\rho}\left(\sum_{j=1}^{2} \frac{\partial}{\partial x_{j}} \int_{b}^{\zeta} \tau_{i j} d x_{3}\right)+\sum_{j=1}^{2} \frac{\partial}{\partial x_{j}}\left(H^{u} U_{j}^{u} U_{i}^{u}+H^{l} U_{j}^{l} U_{i}^{l}\right. \\
& \left.+\int_{d}^{\zeta}\left(u_{i}-U_{i}^{u}\right)\left(u_{j}-U_{j}^{u}\right) d x_{3}+\int_{b}^{d}\left(u_{i}-U_{i}^{l}\right)\left(u_{j}-U_{j}^{l}\right) d x_{3}\right) \\
& =\frac{\partial}{\partial t}\left(H U_{i}\right)-\frac{1}{\rho}\left(\sum_{j=1}^{2} \frac{\partial}{\partial x_{j}} \int_{b}^{\zeta} \tau_{i j} d x_{3}\right)+\sum_{j=1}^{2} \frac{\partial}{\partial x_{j}}\left(H^{u} U_{j}^{u} U_{i}^{u}+H^{l} U_{j}^{l} U_{i}^{l}\right. \\
& \left.+\int_{d}^{\zeta}\left(u_{i} u_{j}-U_{i}^{u} u_{j}-u_{i} U_{j}^{u}+U_{i}^{u} U_{j}^{u}\right) d x_{3}+\int_{b}^{d}\left(u_{i} u_{j}-U_{i}^{l} u_{j}-u_{i} U_{j}^{l}+U_{i}^{l} U_{j}^{l}\right) d x_{3}\right) \\
& =\frac{\partial}{\partial t}\left(H U_{i}\right)-\frac{1}{\rho}\left(\sum_{j=1}^{2} \frac{\partial}{\partial x_{j}} \int_{b}^{\zeta} \tau_{i j} d x_{3}\right)+\sum_{j=1}^{2} \frac{\partial}{\partial x_{j}} \int_{b}^{\zeta} u_{i} u_{j} d x_{3} \\
& =\frac{\partial}{\partial t}\left(H U_{i}\right)-\frac{1}{\rho}\left(\sum_{j=1}^{2} \frac{\partial}{\partial x_{j}} \int_{b}^{\zeta} \tau_{i j} d x_{3}\right)+\sum_{j=1}^{2} \frac{\partial}{\partial x_{j}} \int_{b}^{\zeta}\left(u_{i}-U_{i}\right)\left(u_{j}-U_{j}\right) d x_{3} \\
& +\sum_{j=1}^{2} \frac{\partial}{\partial x_{j}} \int_{b}^{\zeta}\left(u_{i} U_{j}+U_{i} u_{j}-U_{i} U_{j}\right) d x_{3} \\
& =\frac{\partial}{\partial t}\left(H U_{i}\right)+\sum_{j=1}^{2} \frac{\partial}{\partial x_{j}}\left(H U_{j} U_{i}\right)-\frac{1}{\rho} \sum_{j=1}^{2} \frac{\partial}{\partial x_{j}}\left(\int_{b}^{\zeta} \tau_{i j} d x_{3}-\rho \int_{b}^{\zeta} \tilde{u}_{i} \tilde{u}_{j} d x_{3}\right) \\
& =H\left(\frac{\partial}{\partial t} U_{i}+\sum_{j=1}^{2} U_{j} \frac{\partial}{\partial x_{j}} U_{i}\right)-\frac{1}{\rho} \sum_{j=1}^{2} \frac{\partial}{\partial x_{j}}\left(H \tilde{\tau}_{i j}\right) .
\end{aligned}
$$

Finally, it is noted that the above equality is independent of the concrete expressions of the horizontal viscosity terms. 


\section{Appendix 3}

A similar numerical experiment to the simple physical experiment shown in Fig. 4 has been performed. Horizontal sizes of the water tank are $50 \times 10\left[\mathrm{~m}^{2}\right]$. We set $H_{0}^{u}(x)=7.5[\mathrm{~m}]$ and $H_{0}^{l}(x)=2.5[\mathrm{~m}]$. Other similar data as for Mikawa Bay are used for physical values and numerical conditions. Figure 5(a) and Figure 5(b) are stationary contour lines of the water level $\zeta$ and the interface $d$ with a shift of $H_{0}^{u}$ respectively for a sufficient density difference. Figure 5(c) to Figure 5(f) are corresponding contour lines of the interface $d$ with a shift of $H_{0}^{u}$ when the density difference is gradually reduced. The interface becomes unstable as the density difference becomes small. In Fig. 5(d) to Fig. 5(f), computations soon stop because the interface $d$ suddenly rises windward and becomes lower under the wind.

Fig. 5. (a) Contour map of $\zeta\left(\rho^{u}=1000\left[\mathrm{~kg} / \mathrm{m}^{3}\right], \rho^{l}=1200\left[\mathrm{~kg} / \mathrm{m}^{3}\right]\right)$

(b) Contour map of $\left(d+H_{0}^{u}\right) \quad\left(\rho^{u}=1000\left[\mathrm{~kg} / \mathrm{m}^{3}\right], \rho^{l}=1200\left[\mathrm{~kg} / \mathrm{m}^{3}\right]\right)$

(c) Contour map of $\left(d+H_{0}^{u}\right) \quad\left(\rho^{u}=1000\left[\mathrm{~kg} / \mathrm{m}^{3}\right], \rho^{l}=1050\left[\mathrm{~kg} / \mathrm{m}^{3}\right]\right)$

(d) Contour map of $\left(d+H_{0}^{u}\right) \quad\left(\rho^{u}=1000\left[\mathrm{~kg} / \mathrm{m}^{3}\right], \rho^{l}=1005\left[\mathrm{~kg} / \mathrm{m}^{3}\right]\right)$

(e) Contour map of $\left(d+H_{0}^{u}\right) \quad\left(\rho^{u}=1000\left[\mathrm{~kg} / \mathrm{m}^{3}\right], \rho^{l}=1001\left[\mathrm{~kg} / \mathrm{m}^{3}\right]\right)$

(f) Contour map of $\left(d+H_{0}^{u}\right) \quad\left(\rho^{u}=1000\left[\mathrm{~kg} / \mathrm{m}^{3}\right], \rho^{l}=1000\left[\mathrm{~kg} / \mathrm{m}^{3}\right]\right)$ 


\section{$\underline{\text { Figure Legends }}$}

Fig.1. 2 layer model

Fig.2. (a) Contour map of $\zeta$ after 75 hours (Cond. 1)

(b) Transition of $\zeta$ (Cond. 1)

(c) Transition of $\left(d+H_{0}^{u}\right)$ (Cond. 1)

(d) Transition of $\left(d+H_{0}^{u}\right)$ (Cond. 1 and Cond. 2)

Fig.3. (a) Contour map of $\zeta$ after 55 hours (Cond. 3)

(b) Transmition of $\zeta$ (Cond. 3)

(c) Contour map of $\left(d+H_{0}^{u}\right)$ after 55 hours (Cond. 3)

(d) Transmition of $\left(d+H_{0}^{u}\right)$ (Cond. 3)

(e) Contour map of $\zeta$ and $\left(d+H_{0}^{u}\right)$ after 380 hours (Cond. 4)

(f) Transmition of $\zeta$ and $\left(d+H_{0}^{u}\right)$ (Cond. 4)

(g) Velocity vectors of upper layer after 380 hours (Cond. 4)

(h) Velocity vectors of lower layer after 380 hours (Cond. 4)

Fig.4. An experimental two layer flow

Fig.5. (a) Contour map of $\zeta\left(\rho^{u}=1000\left[\mathrm{~kg} / \mathrm{m}^{3}\right], \rho^{l}=1200\left[\mathrm{~kg} / \mathrm{m}^{3}\right]\right)$

(b) Contour map of $\left(d+H_{0}^{u}\right) \quad\left(\rho^{u}=1000\left[\mathrm{~kg}^{\prime} \mathrm{m}^{3}\right], \rho^{l}=1200\left[\mathrm{~kg} / \mathrm{m}^{3}\right]\right)$

(c) Contour map of $\left(d+H_{0}^{u}\right) \quad\left(\rho^{u}=1000\left[\mathrm{~kg} / \mathrm{m}^{3}\right], \rho^{l}=1050\left[\mathrm{~kg} / \mathrm{m}^{3}\right]\right)$

(d) Contour map of $\left(d+H_{0}^{u}\right) \quad\left(\rho^{u}=1000\left[\mathrm{~kg} / \mathrm{m}^{3}\right], \rho^{l}=1005\left[\mathrm{~kg} / \mathrm{m}^{3}\right]\right)$ 
(e) Contour map of $\left(d+H_{0}^{u}\right) \quad\left(\rho^{u}=1000\left[\mathrm{~kg} / \mathrm{m}^{3}\right], \rho^{l}=1001\left[\mathrm{~kg} / \mathrm{m}^{3}\right]\right)$

(f) Contour map of $\left(d+H_{0}^{u}\right) \quad\left(\rho^{u}=1000\left[\mathrm{~kg} / \mathrm{m}^{3}\right], \rho^{l}=1000\left[\mathrm{~kg} / \mathrm{m}^{3}\right]\right)$ 


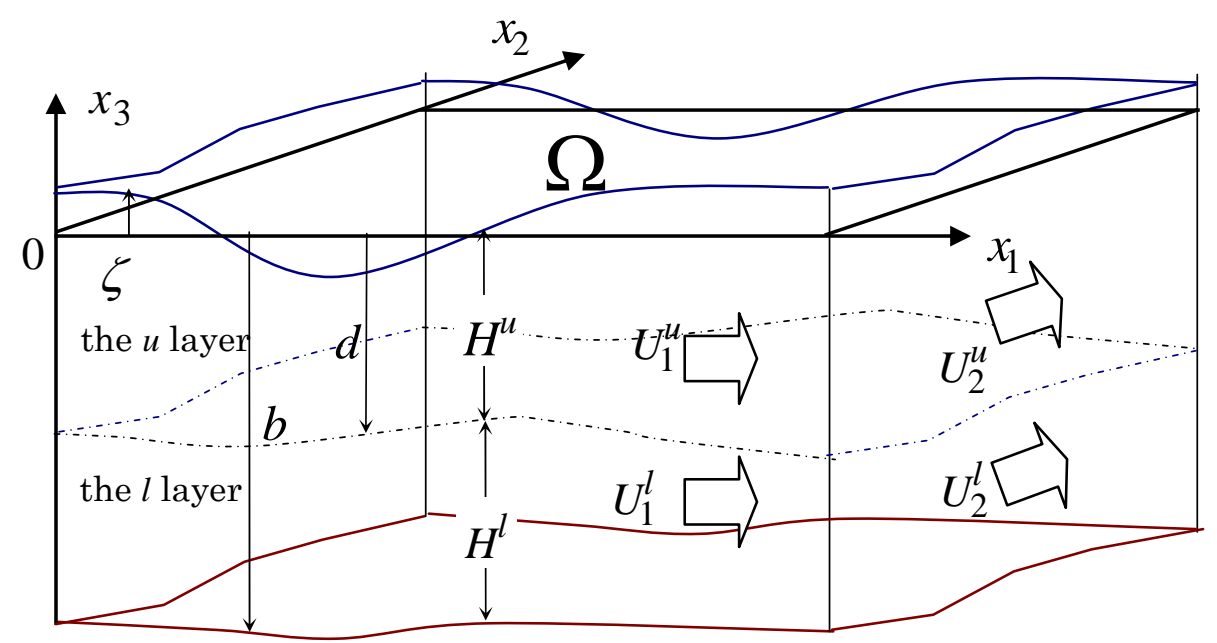

Fig. 1. 2 layer model 
Fig. 2. (a) Contour map of $\zeta$ after 75 hours (Cond. 1)

(b) Transition of $\zeta$ (Cond. 1)

(c) Transition of $\left(d+H_{0}^{u}\right)$ (Cond. 1)

(d) Transition of $\left(d+H_{0}^{u}\right)$ (Cond. 1 and Cond. 2) 


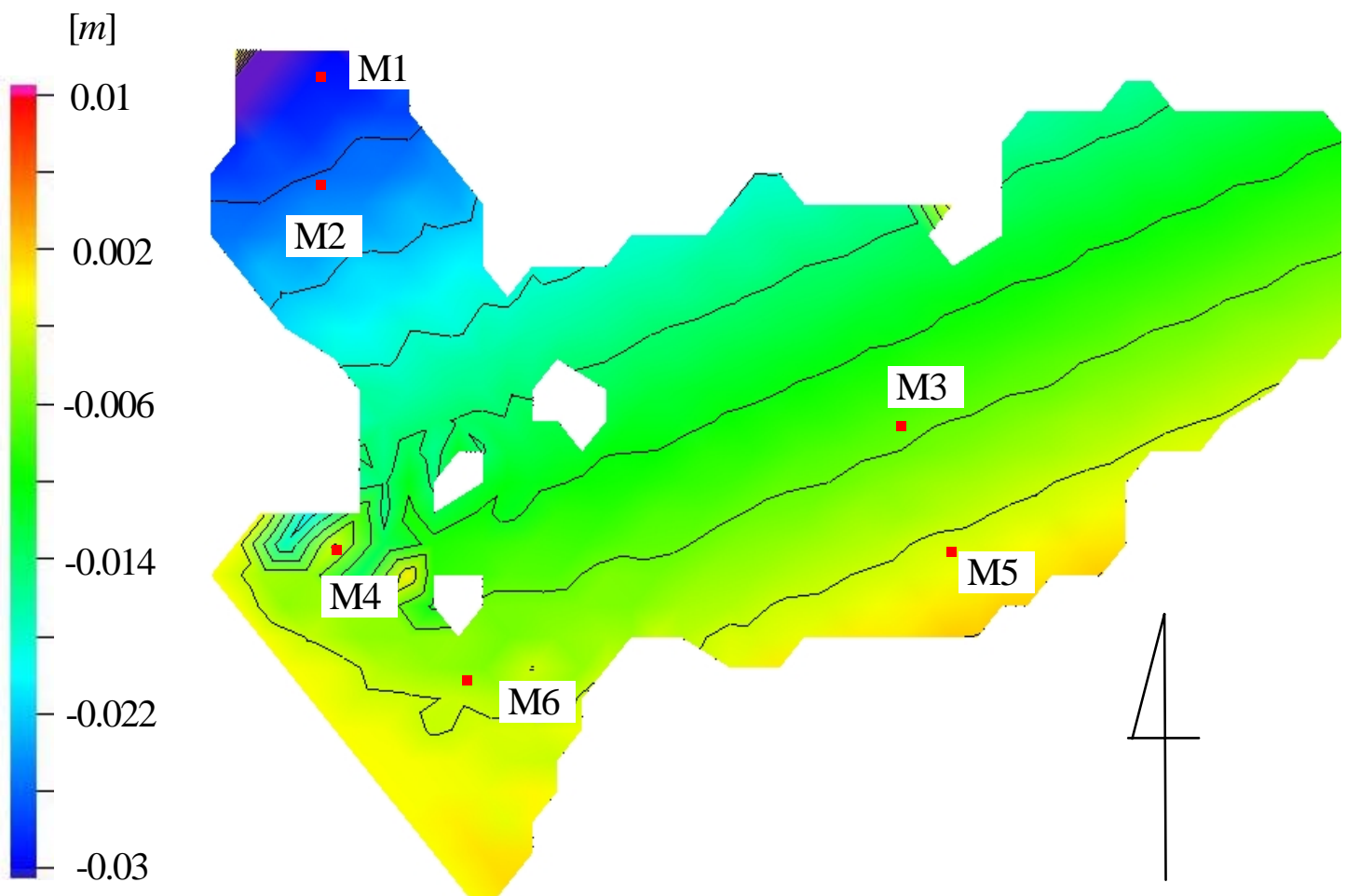

(a)

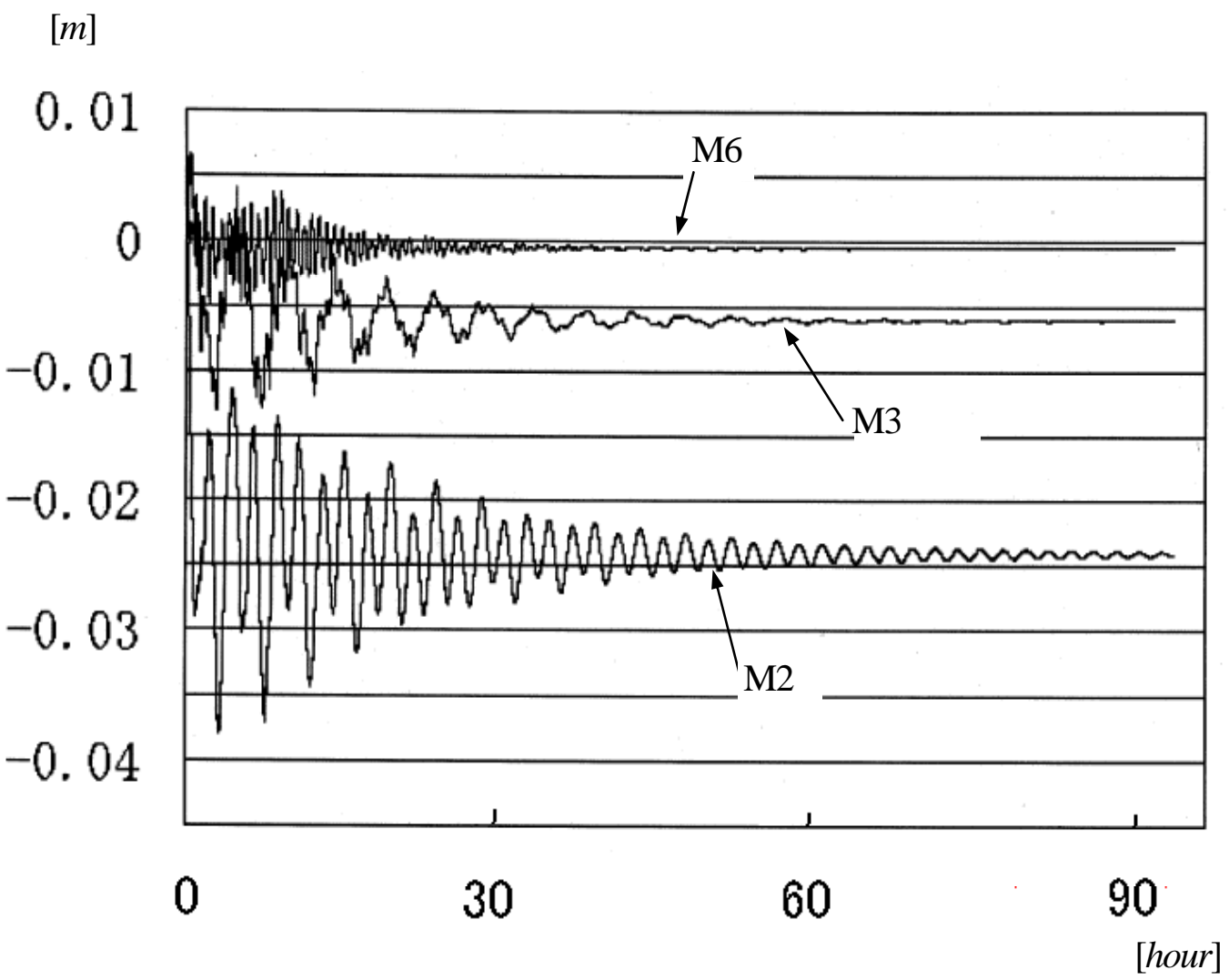

(b) 


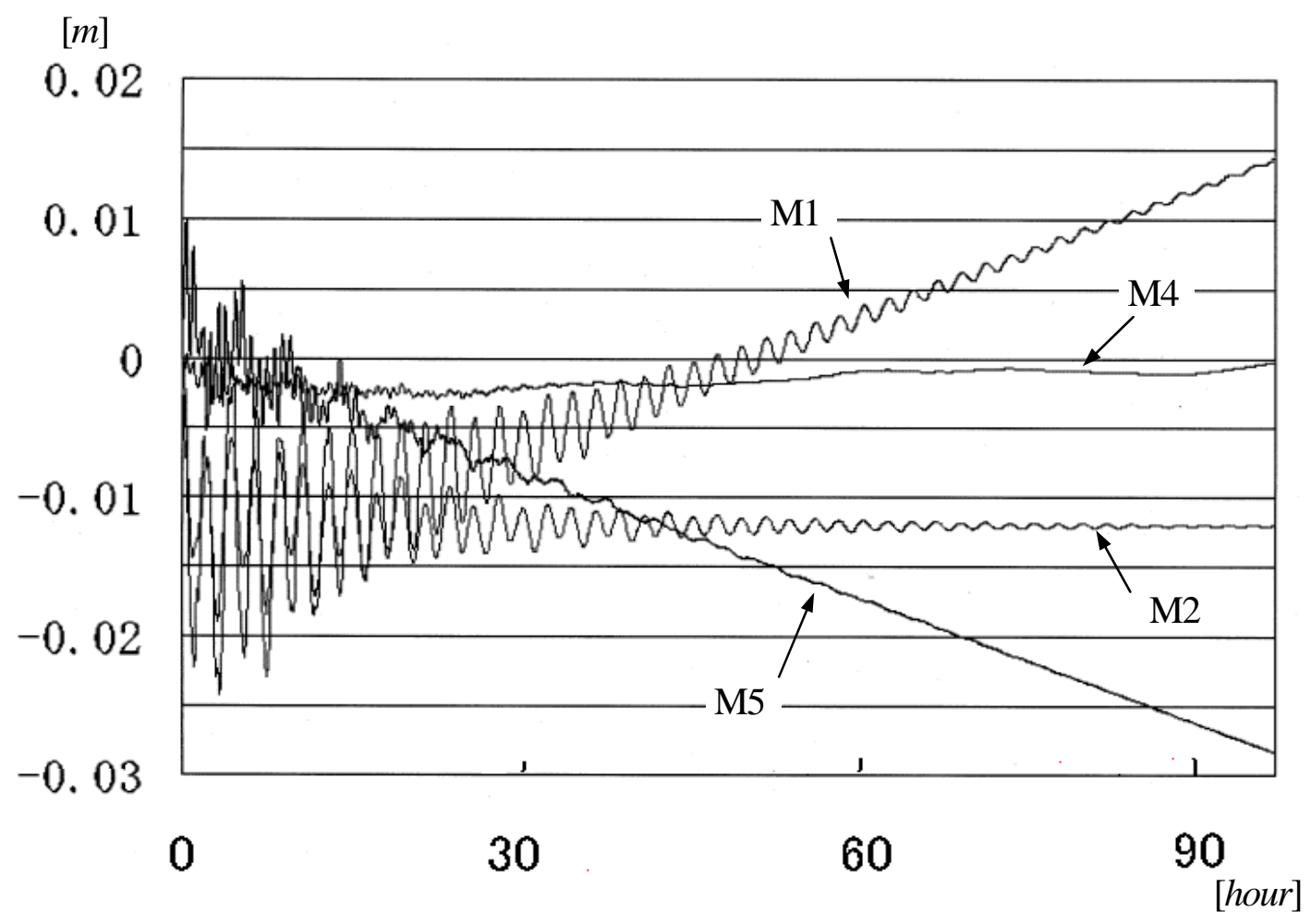

(c)

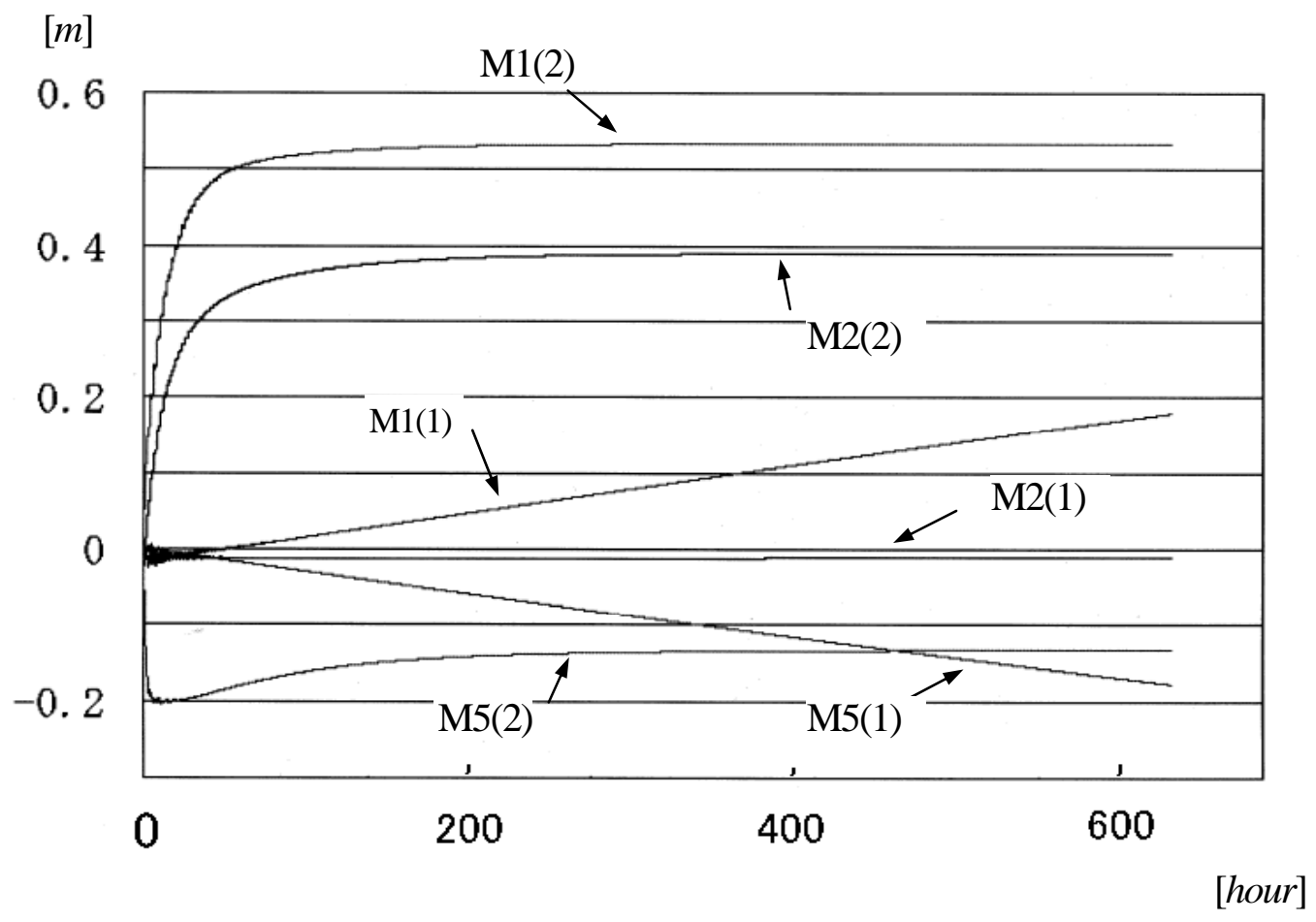

(d) 
Fig. 3. (a) Contour map of $\zeta$ after 55 hours (Cond. 3)

(b) Transmition of $\zeta$ (Cond. 3)

(c) Contour map of $\left(d+H_{0}^{u}\right)$ after 55 hours (Cond. 3)

(d) Transmition of $\left(d+H_{0}^{u}\right)$ (Cond. 3)

(e) Contour map of $\zeta$ and $\left(d+H_{0}^{u}\right)$ after 380 hours (Cond. 4)

(f) Transmition of $\zeta$ and $\left(d+H_{0}^{u}\right)$ (Cond. 4)

(g) Velocity vectors of upper layer after 380 hours (Cond. 4)

(h) Velocity vectors of lower layer after 380 hours (Cond. 4) 


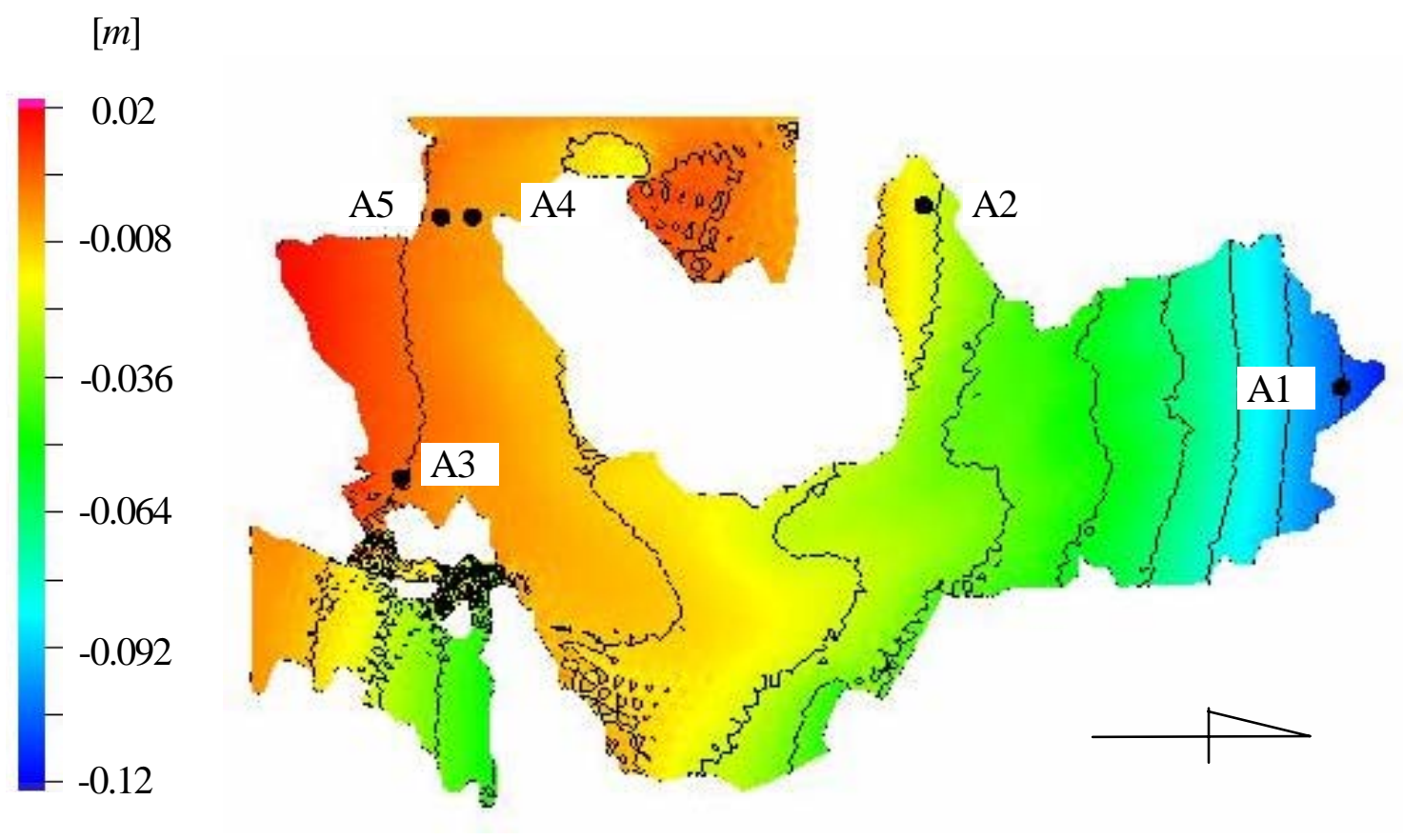

(a)

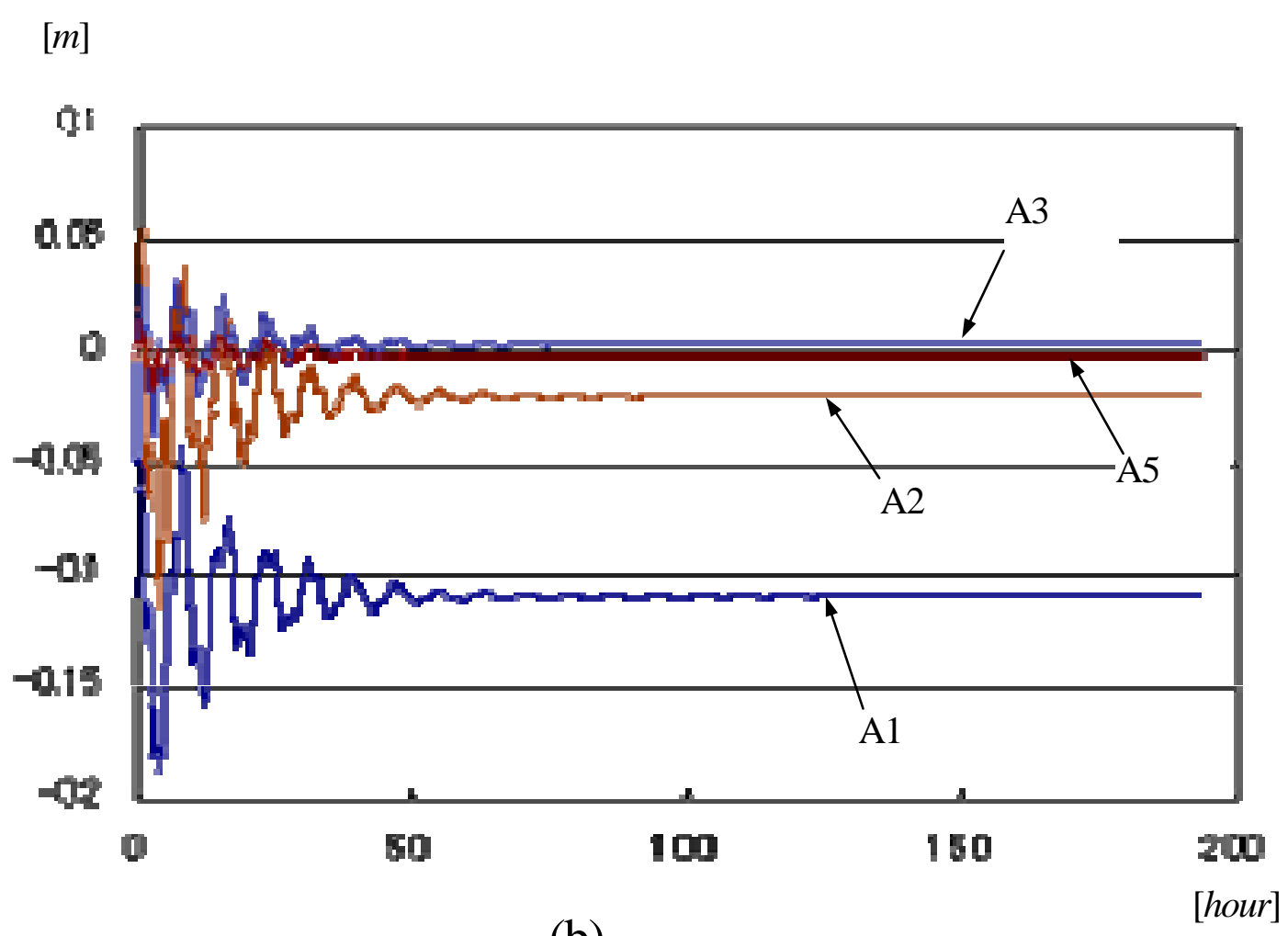

(b) 

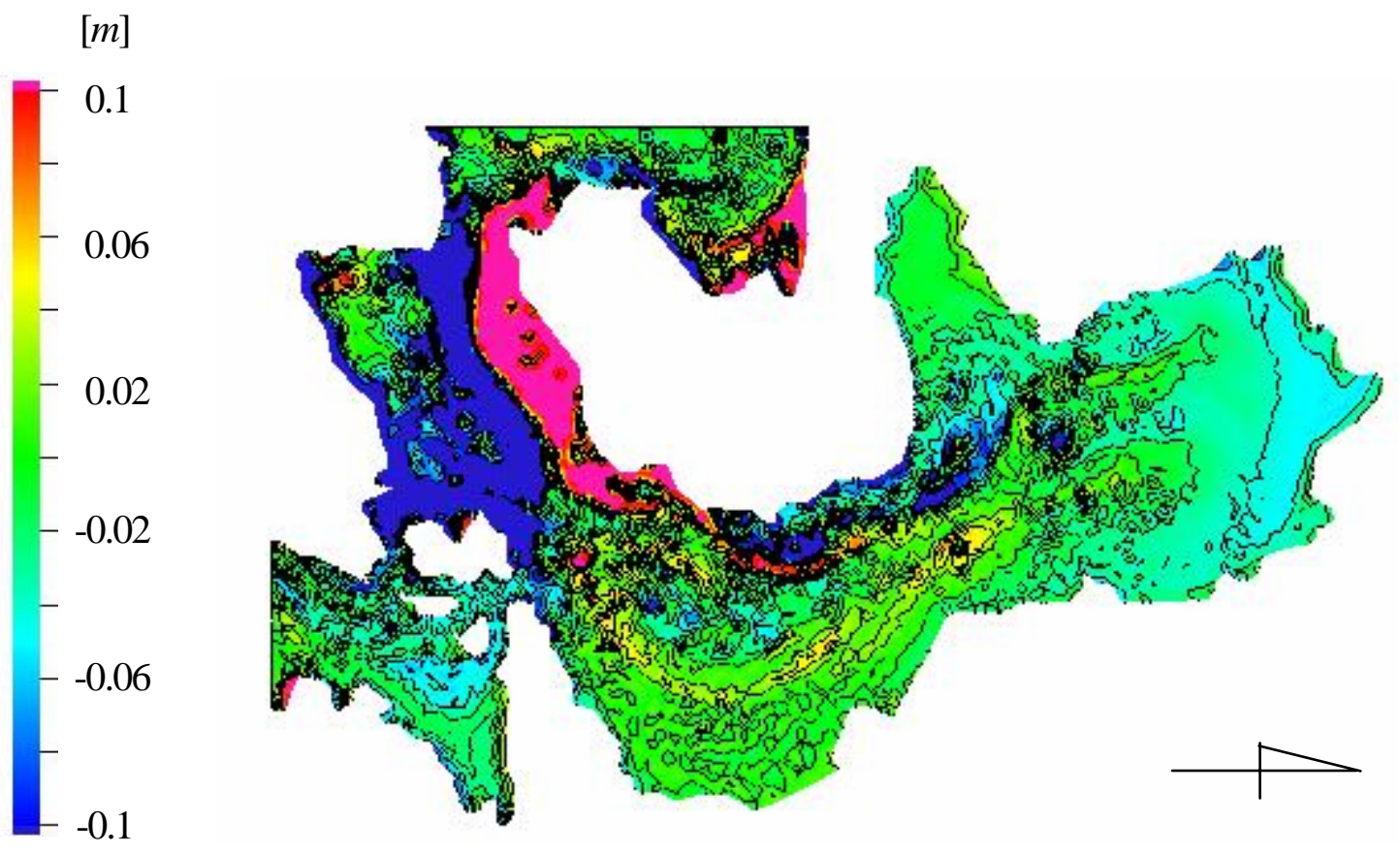

(c)

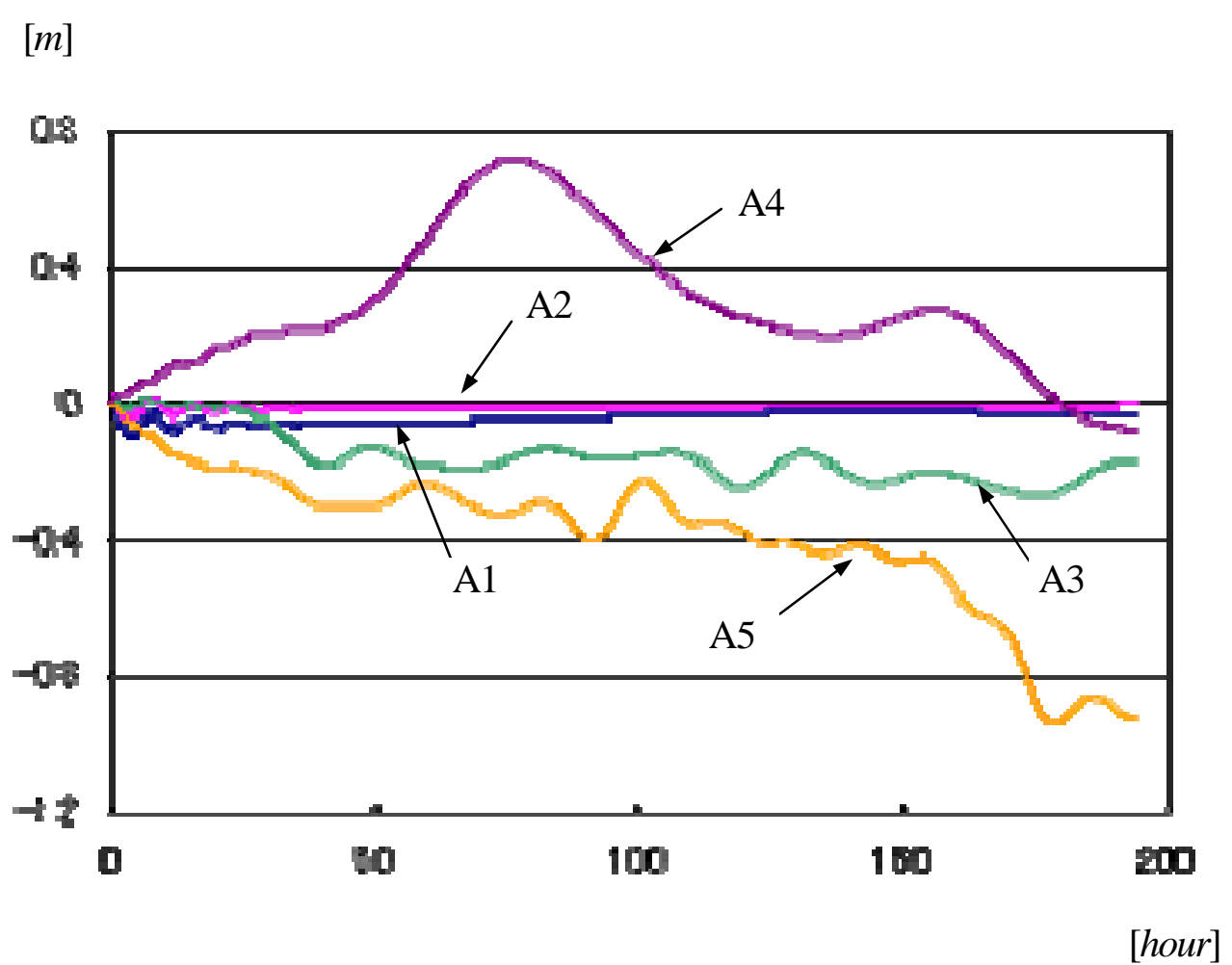

(d) 


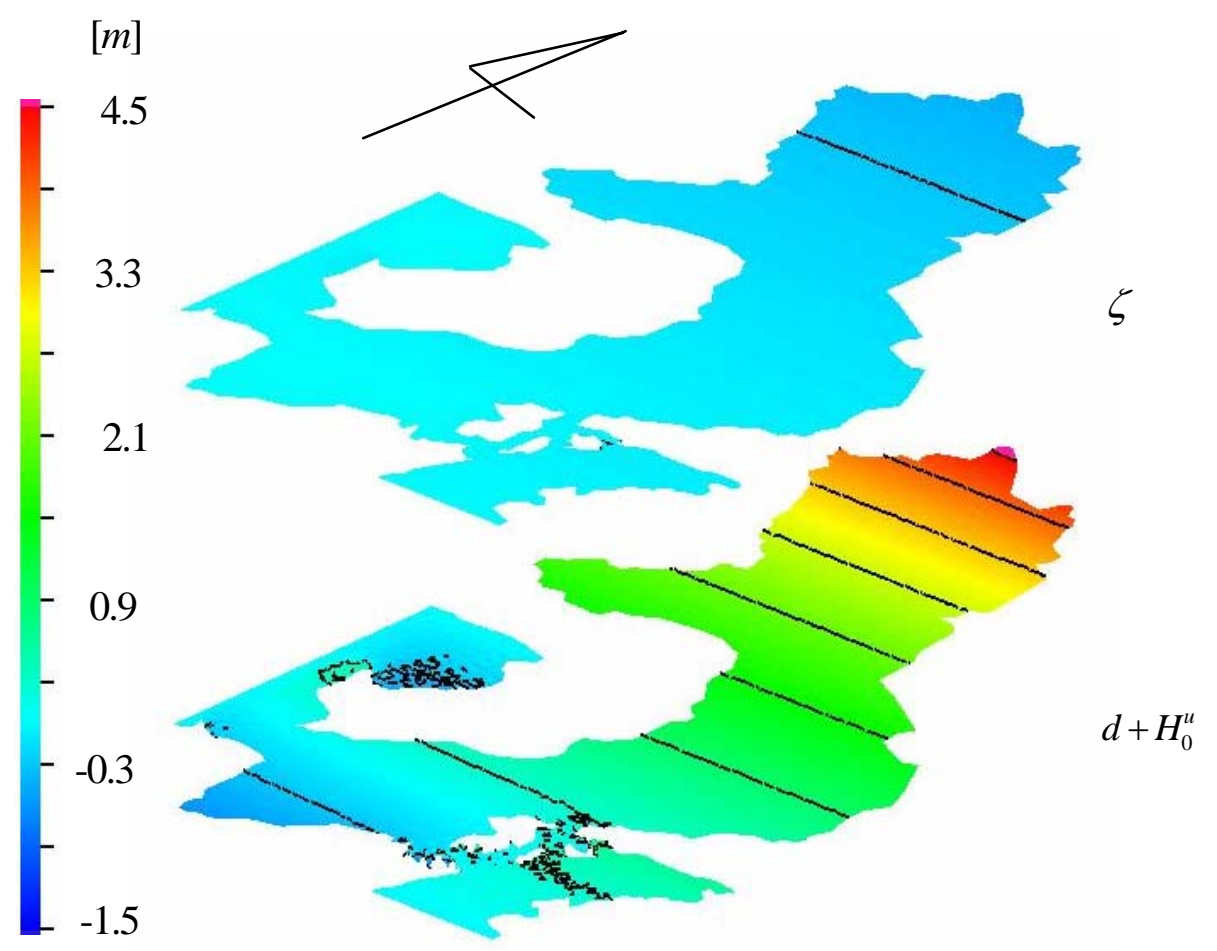

(e)

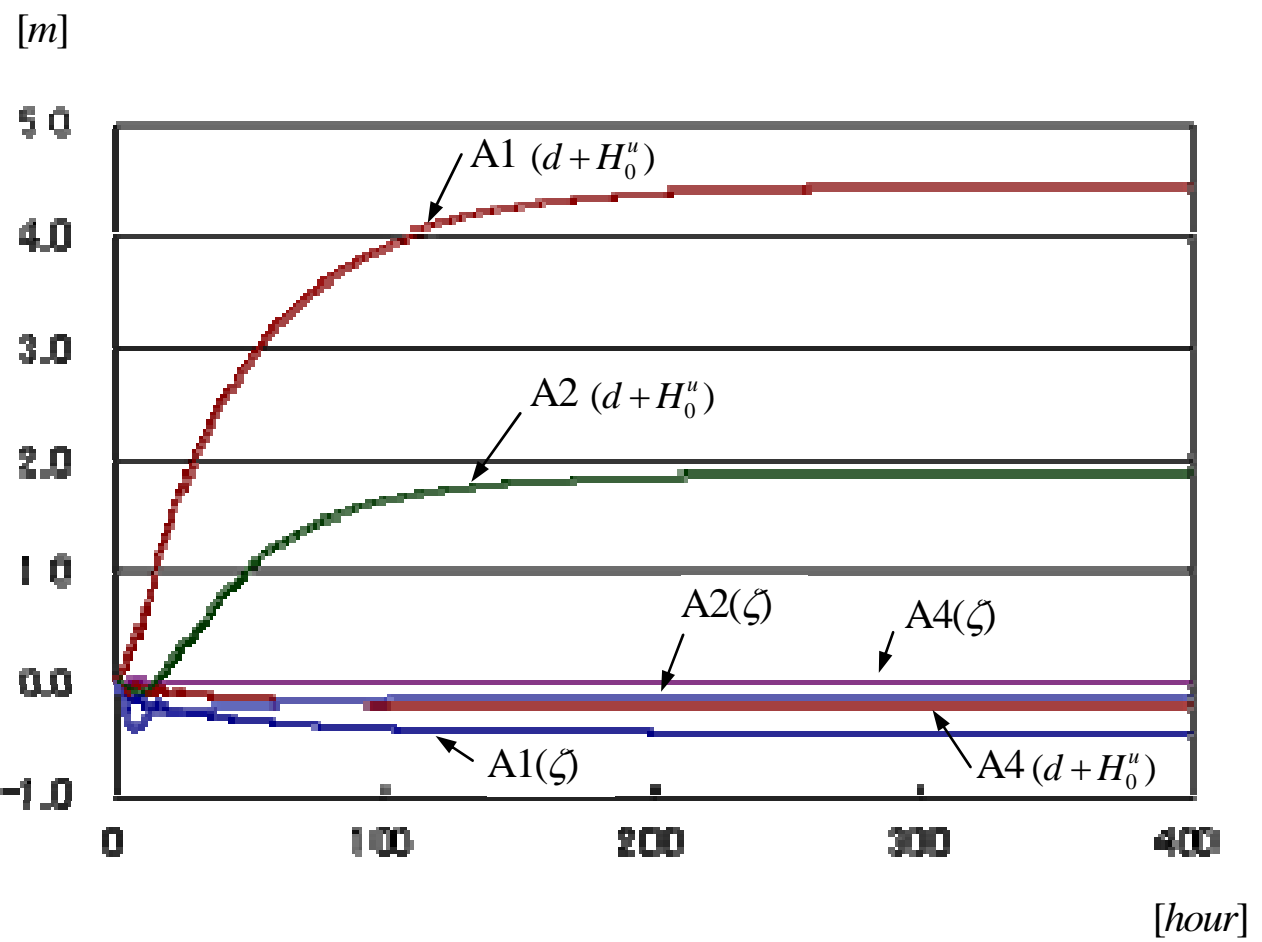

(f) 

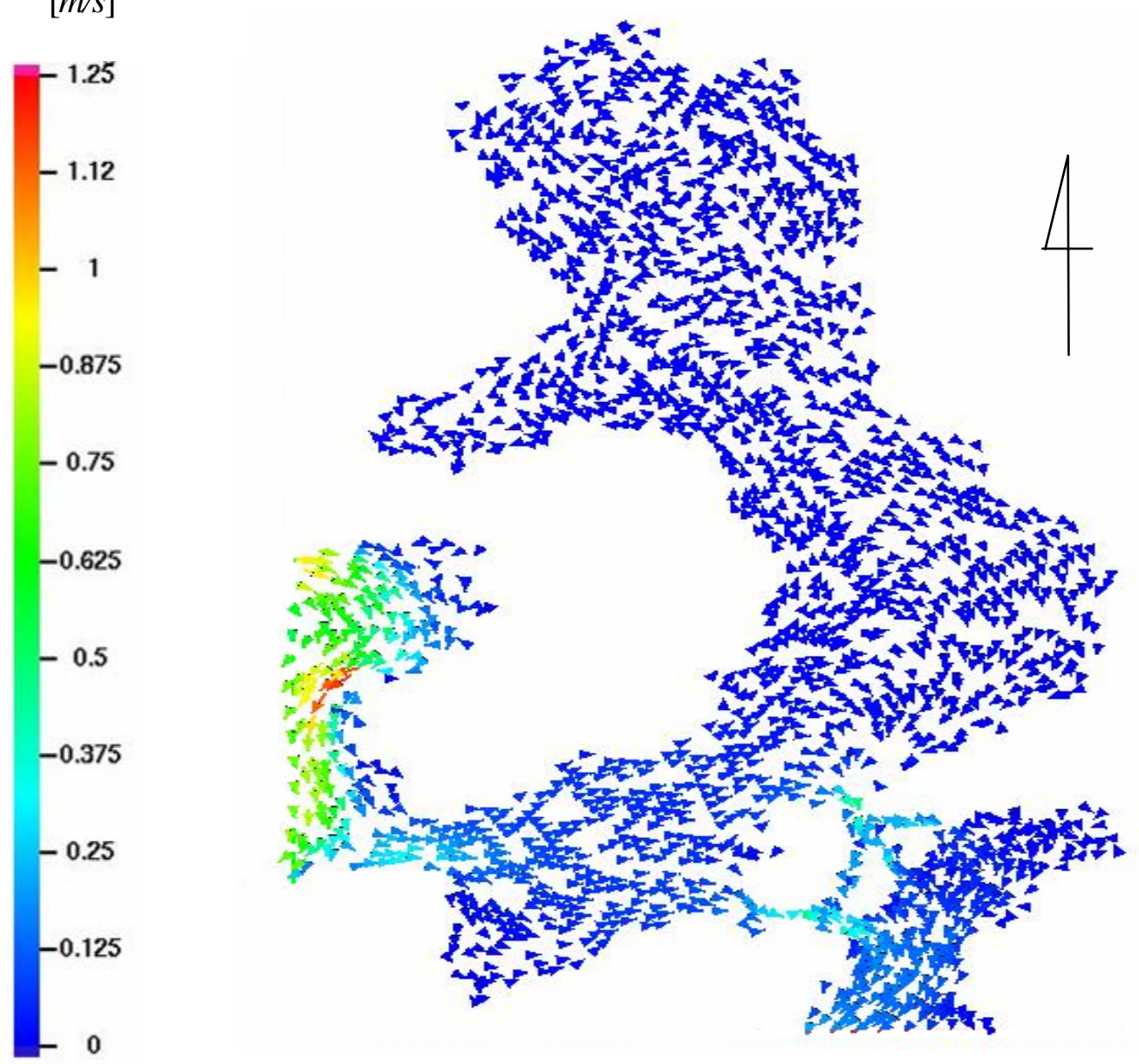

(g)

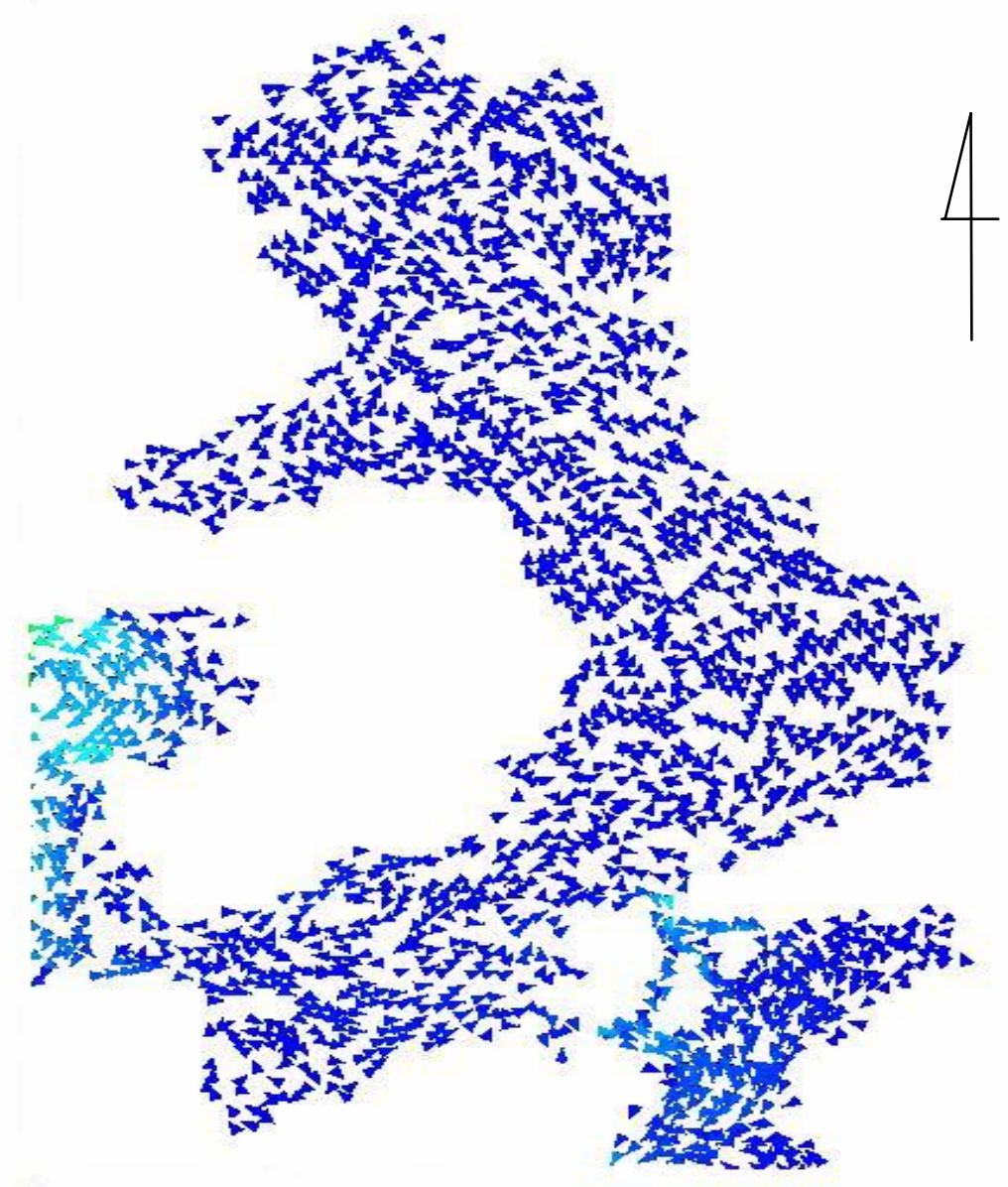

(h) 


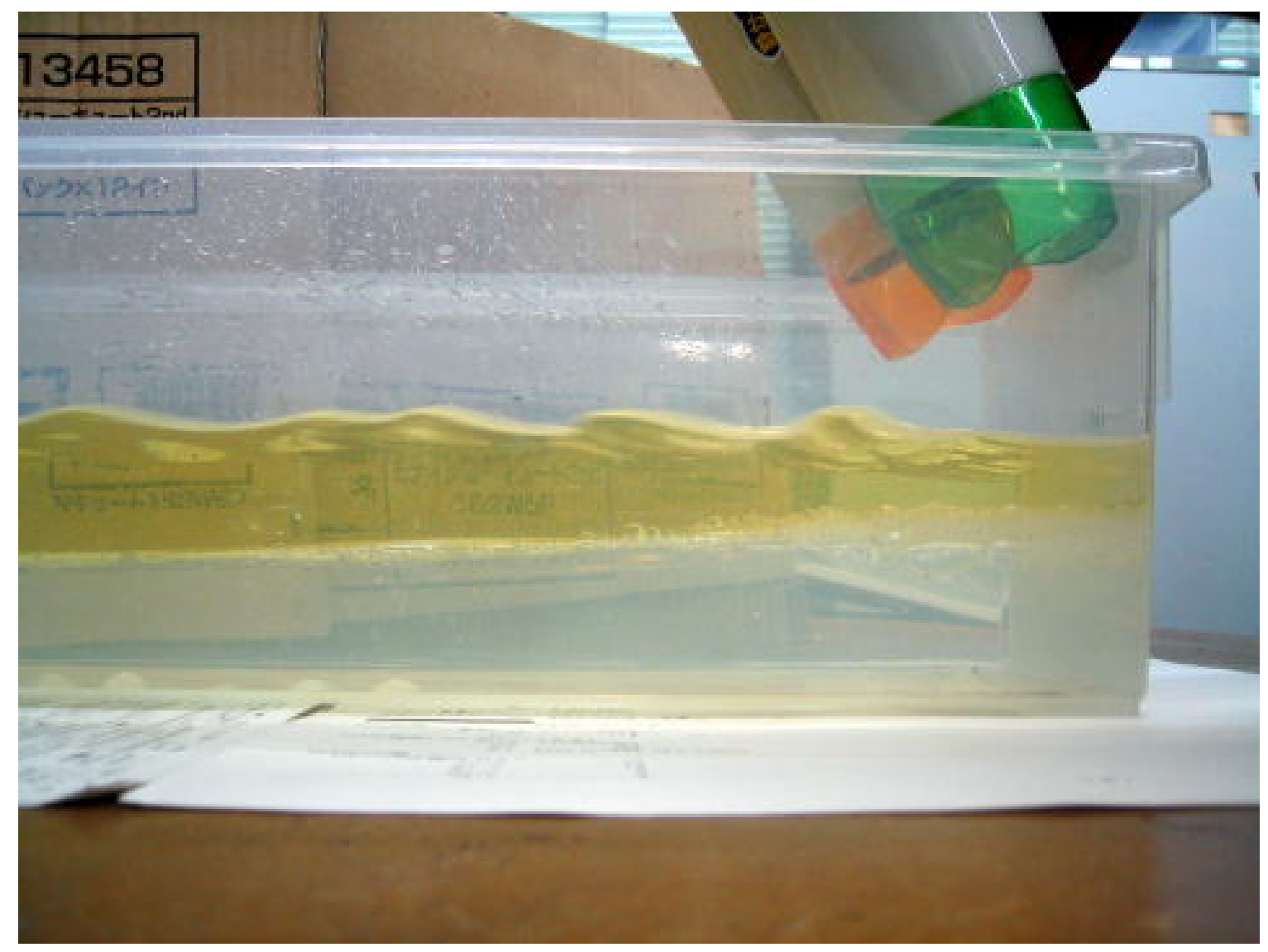

Fig. 4. An experimental two layer flow 
Fig. 5. (a) Contour map of $\zeta\left(\rho^{u}=1000\left[\mathrm{~kg} / \mathrm{m}^{3}\right], \rho^{l}=1200\left[\mathrm{~kg} / \mathrm{m}^{3}\right]\right)$

(b) Contour map of $\left(d+H_{0}^{u}\right) \quad\left(\rho^{u}=1000\left[\mathrm{~kg} / \mathrm{m}^{3}\right], \rho^{l}=1200\left[\mathrm{~kg} / \mathrm{m}^{3}\right]\right)$

(c) Contour map of $\left(d+H_{0}^{u}\right) \quad\left(\rho^{u}=1000\left[\mathrm{~kg} / \mathrm{m}^{3}\right], \rho^{l}=1050\left[\mathrm{~kg} / \mathrm{m}^{3}\right]\right)$

(d) Contour map of $\left(d+H_{0}^{u}\right) \quad\left(\rho^{u}=1000\left[\mathrm{~kg} / \mathrm{m}^{3}\right], \rho^{l}=1005\left[\mathrm{~kg} / \mathrm{m}^{3}\right]\right)$

(e) Contour map of $\left(d+H_{0}^{u}\right) \quad\left(\rho^{u}=1000\left[\mathrm{~kg} / \mathrm{m}^{3}\right], \rho^{l}=1001\left[\mathrm{~kg} / \mathrm{m}^{3}\right]\right)$

(f) Contour map of $\left(d+H_{0}^{u}\right) \quad\left(\rho^{u}=1000\left[\mathrm{~kg} / \mathrm{m}^{3}\right], \rho^{l}=1000\left[\mathrm{~kg} / \mathrm{m}^{3}\right]\right)$ 


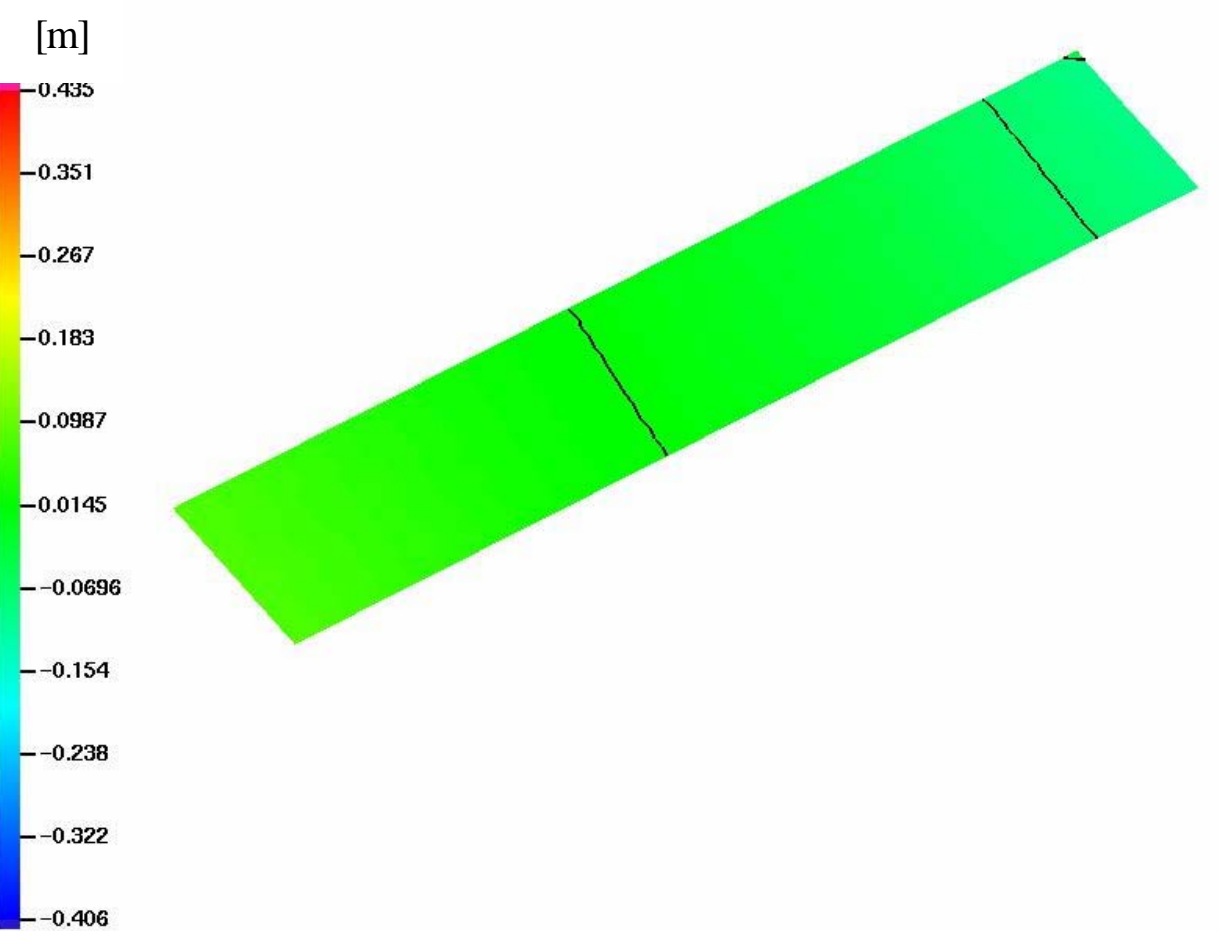

(a)

[m]
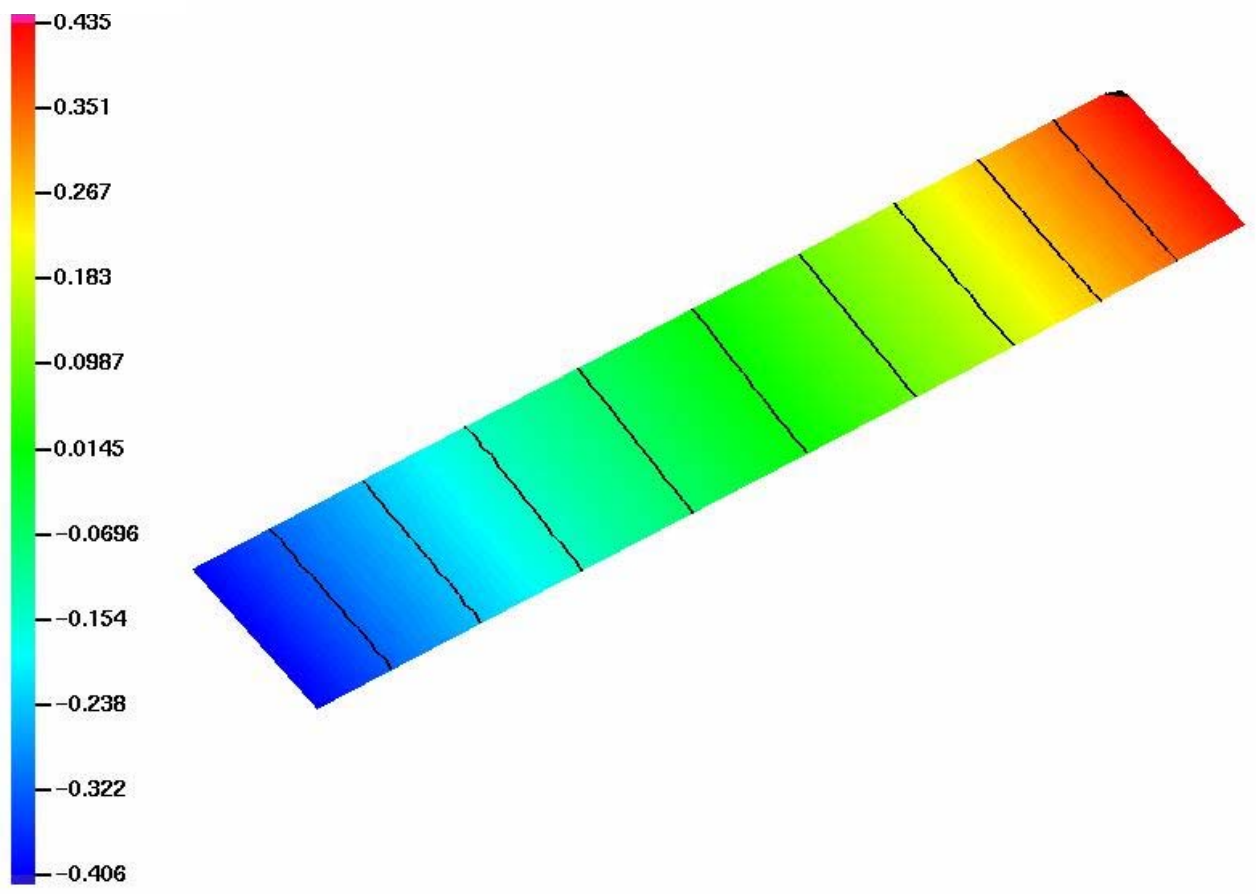

(b) 


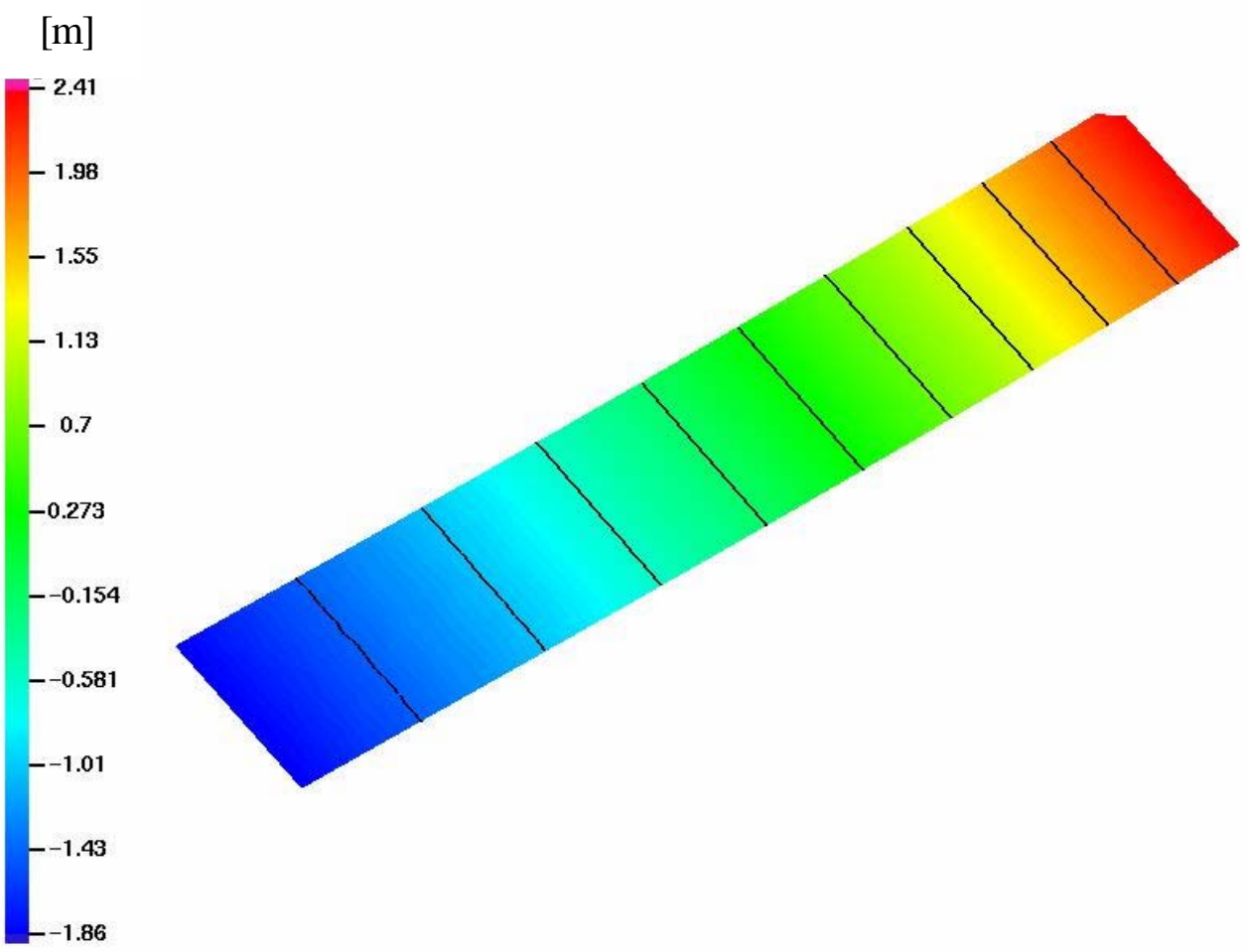

(c)
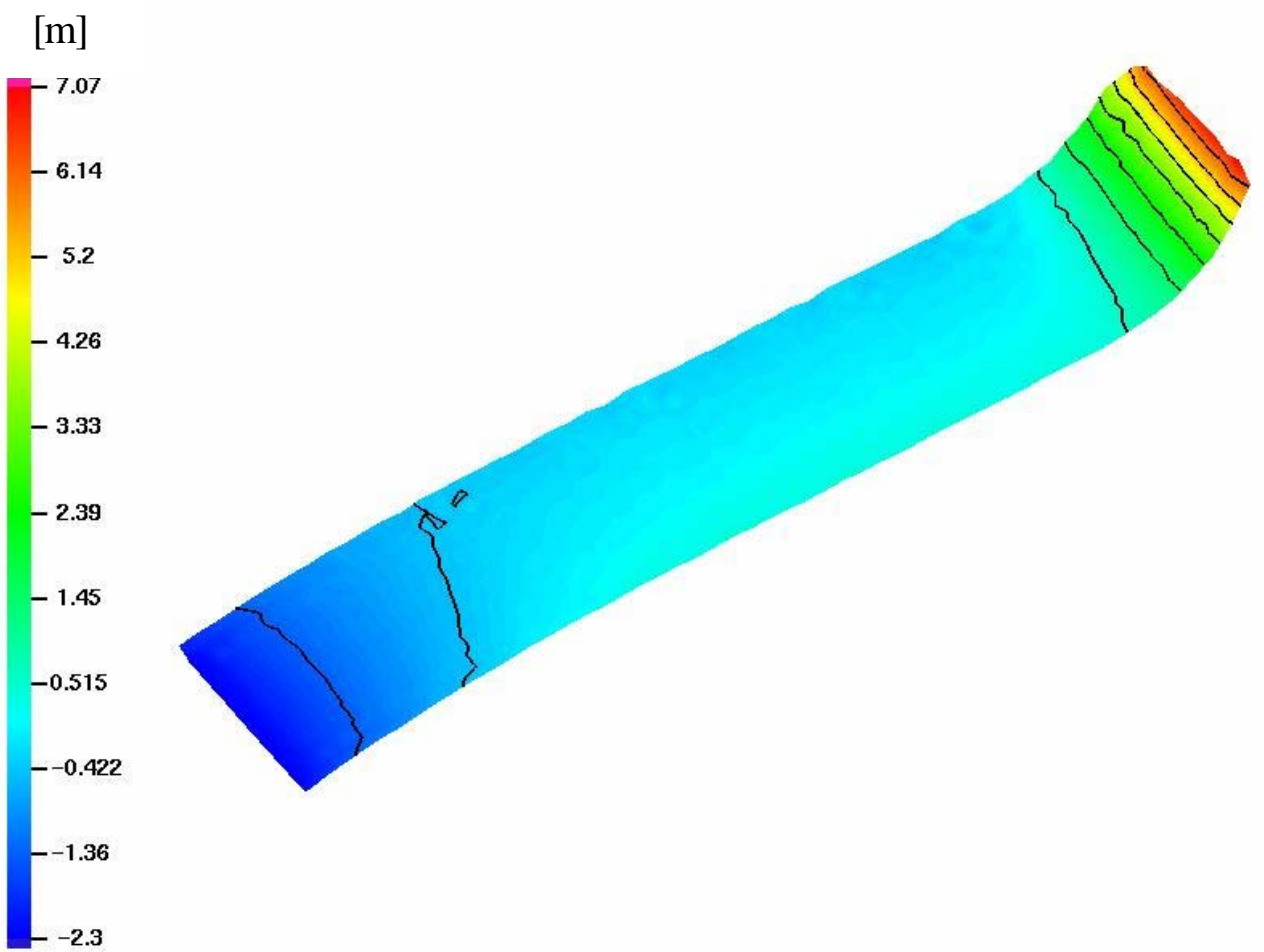

(d) 


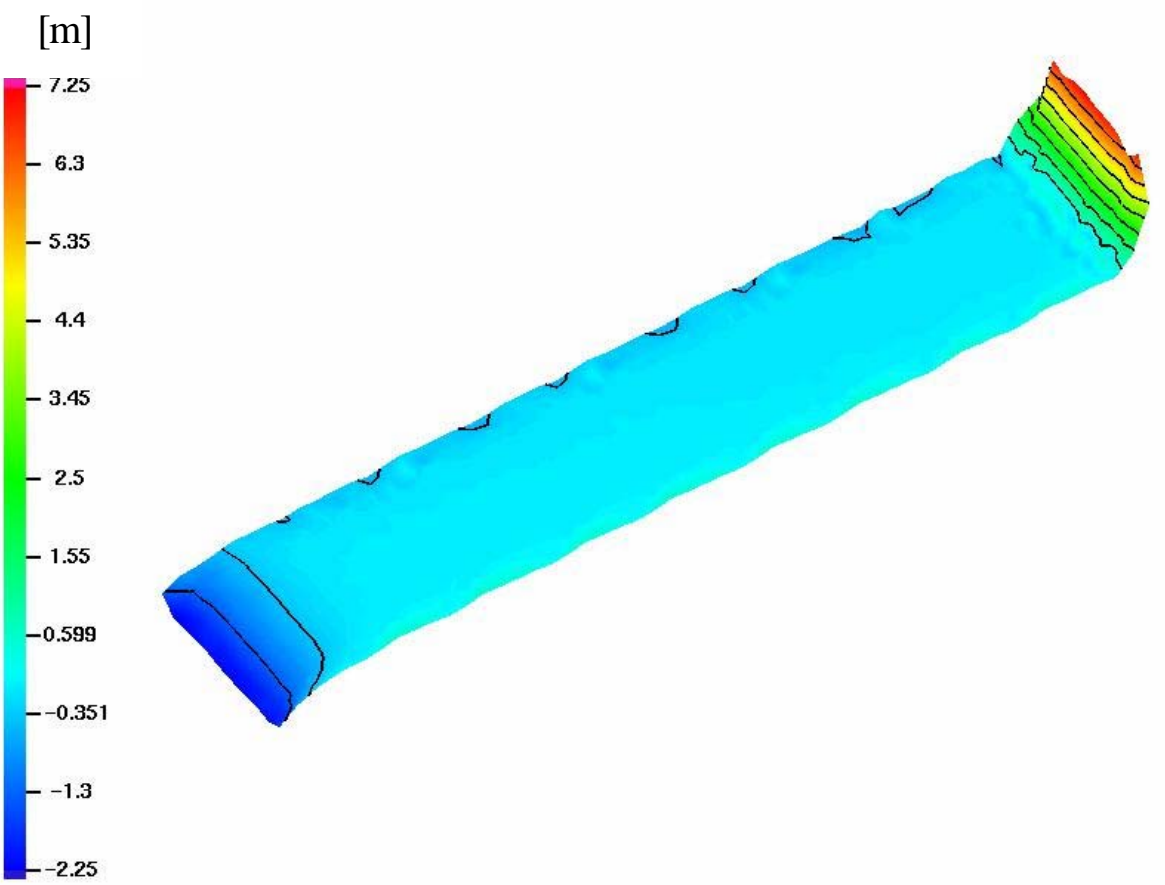

(e)
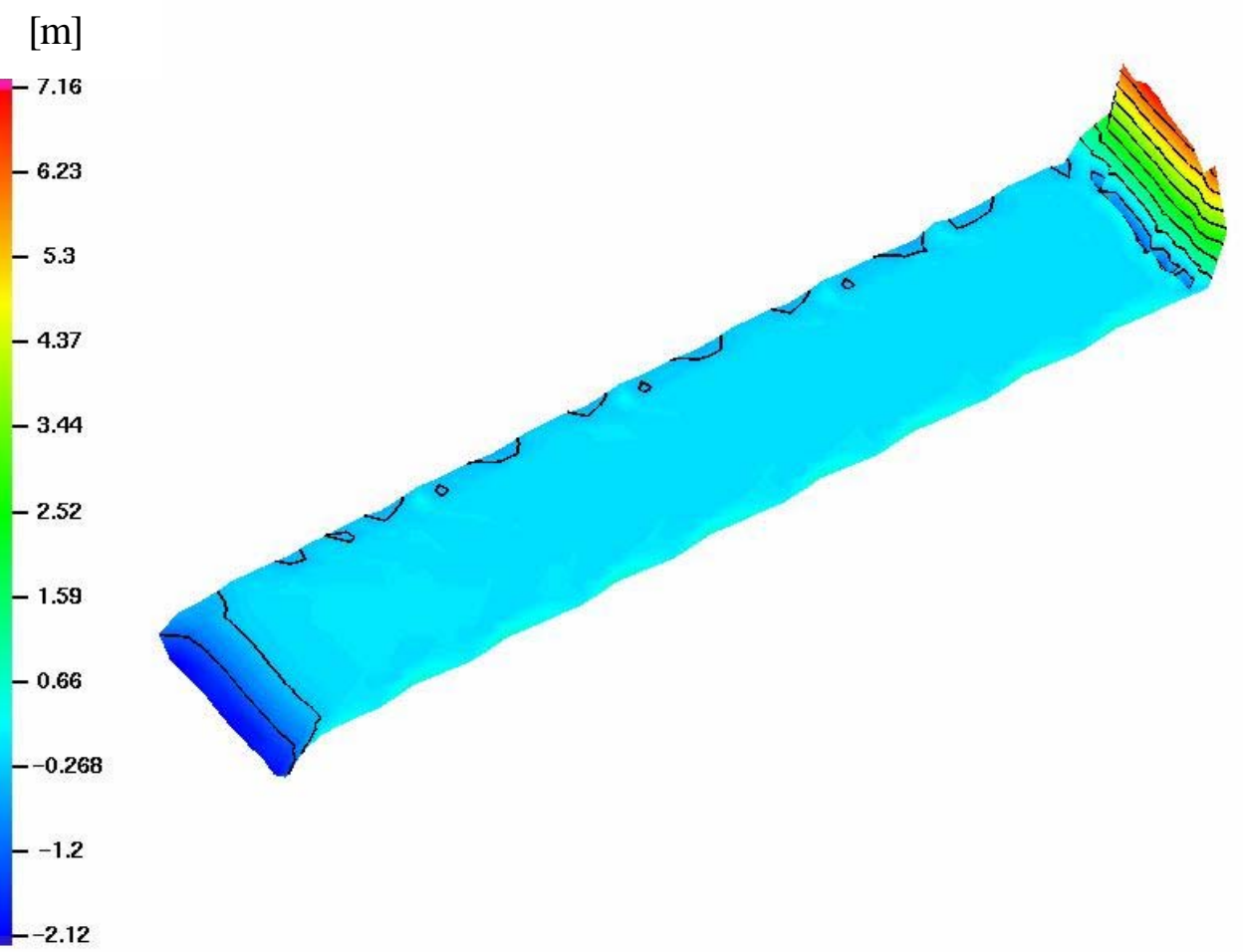

(f) 